\title{
Polarized neutron diffraction
}

\author{
E. Ressouche \\ SPSMS, UMR-E CEA/UJF-Grenoble 1, INAC, 38054 Grenoble, France
}

\begin{abstract}
This lecture addresses the different applications of polarized neutron diffraction, with or without subsequent polarization analysis, to the determination of magnetic structures, to the separation of magnetic and nuclear contributions and to magnetization distributions mapping. The first sections introduce some of the fundamental equations. Then the problems of magnetic domains, encountered in crystals and of particular importance as soon as polarized neutrons are concerned, are presented. The final sections discuss some of the different ways to use polarized neutron and are illustrated by examples.
\end{abstract}

\section{PREAMBLE}

Neutron scattering has progressed over the last sixty years to become an invaluable tool to probe experimentally condensed matter. As far as magnetism is concerned, this technique has been recognized from the early days as unique. The most widespread use of this tool is of course the determination of magnetic structures using unpolarized beams, that is the determination of the directions in which moments point in a magnetically ordered material. Since the first experiment on $\mathrm{MnO}$ by Shull and Smart in 1949 [1] which was an experimental proof of the antiferromagnetism predicted by Néel, thousands of structure determinations have been reported and have revealed much more complicated magnetic arrangements than the simple ferromagnetic or antiferromagnetic cases. The comprehension of the stability of these new structures was and is still at the origin of numerous theoretical developments, leaving the study of magnetic structures completely open.

From a theoretical point of view, the neutron spin and the possibility of using polarized neutron beam was taken into account from the very beginning [2-6]. Complete equations of polarization analysis in a neutron scattering experiment were derived as early as 1963 [7, 8]. But in comparison to those theoretical advances, experimental possibilities were much longer to appear. In 1959 Nathans et al. demonstrated the huge sensitivity of polarized neutrons to weak magnetic signals, allowing very precise magnetization distribution maps to be drawn [9]. Then it took ten years to Moon, Riste and Koehler to operate in Oak Ridge the first triple axis machine with both incident polarized neutrons and longitudinal polarization analysis capabilities, giving access to many new pieces of information [10]. Another twenty years were necessary until Tasset et al. generalized longitudinal polarization analysis to a completely spherical one [11]. During this time, Maleyev also showed that polarization of neutron beams could allow very precise measurements of the energy exchange in a scattering experiment, leading to the development of the spin echo technique by Mezei [12] and to the numerous applications this technique has today.

This is an Open Access article distributed under the terms of the Creative Commons Attribution License 4.0, which permits unrestricted use, distribution, and reproduction in any medium, provided the original work is properly cited. 


\section{Collection SFN}

Like it is often the case, progresses in the field are due to technical developments, in particular in the ways to produce polarized neutrons. Those progress, aiming at increase the flux at sample position, are such that nowadays, it is possible to equip most of the existing spectrometers with a polarized neutron option at minimum expenses, and therefore make this technique accessible to more and more users. In front of all the developments performed over the last sixty years, a complete description of the different aspects of polarized neutron diffraction would be a task too long and is out of the scope of this chapter. The aim of this contribution is to present the different techniques to a potential user, to introduce some of the fundamental aspects and to convince the reader on how useful neutron scattering could be to solve a particular problem he (or she) may have.

\section{POLARIZED NEUTRONS: WHAT DOES IT MEAN?}

Talking about neutron polarization could be abusive. Indeed, one single neutron is not polarized. What can be polarized is a beam of neutrons. Each individual neutron carries a spin $\mathbf{s}$, an intrinsic angular momentum with a quantum number $1 / 2$. This value $s=1 / 2$ means that the eigenvalue of the operator $\mathbf{s}^{2}$ is $s(s+1) \hbar^{2}=3 / 4 \hbar^{2}$ and that the eigenvalues of the operator $s_{z}$, the projection of the operator $\mathbf{s}$ on the $\mathrm{Oz}$ axis, are $m_{s}= \pm 1 / 2 \hbar$, whatever the chosen $\mathrm{Oz}$ axis. A polarized beam means that all the neutrons of the beam are in one of these two eigenstates.

$\mathbf{s}$ is a vectorial operator, with 3 components $s_{x}, s_{y}$ and $s_{z}$, which can be expressed in terms of Pauli matrices in the 2 dimensional Hilbert spaces for spin $1 / 2$. It is convenient to define the operator $\boldsymbol{\sigma}=2 \mathrm{~s} / \hbar$. Then one can write:

$$
\sigma_{x}=\left(\begin{array}{ll}
0 & 1 \\
1 & 0
\end{array}\right), \quad \sigma_{y}=\left(\begin{array}{rr}
0 & -i \\
i & 0
\end{array}\right), \quad \sigma_{z}=\left(\begin{array}{rr}
1 & 0 \\
0 & -1
\end{array}\right) .
$$

For one neutron $\mathrm{j}$ in the beam, one can define a vector $\mathbf{p}_{\mathbf{j}}$ from the 3 expectations values of the 3 components of $\sigma$ :

$$
\mathbf{p}_{\mathbf{j}}=\langle\boldsymbol{\sigma}\rangle=\left(\begin{array}{c}
\left\langle\sigma_{x}\right\rangle \\
\left\langle\sigma_{y}\right\rangle \\
\left\langle\sigma_{z}\right\rangle
\end{array}\right) .
$$

The polarization of the neutron beam is the average of the individual polarizations of all the neutrons in the beam:

$$
\mathbf{P}=\frac{1}{N} \sum_{j} \mathbf{p}_{\mathbf{j}} .
$$

It should be noticed that with such a definition the polarization of the beam $\mathbf{P}$ is a classical vector and it is therefore possible to measure simultaneously its 3 components. In a particular direction $\alpha$, the component $P_{\alpha}$ can be written:

$$
P_{\alpha}=\frac{n^{+}-n^{-}}{n^{+}+n^{-}}
$$

where $n^{+}$(resp. $n^{-}$) is the number of neutrons in the $+1 / 2($ resp. $-1 / 2)$ eigenstate. With such a definition, one has obviously:

$$
0 \leq|\mathbf{P}| \leq 1
$$

Associated to its spin, the neutron carries a magnetic moment:

$$
\boldsymbol{\mu}_{n}=\gamma \mu_{N} \boldsymbol{\sigma}=\gamma_{L} \boldsymbol{S}
$$


with $\gamma_{L}=(2 / \hbar) \gamma \mu_{N}, \mu_{N}$ being the nuclear Bohr magneton and $\gamma=-1.913$ the value of the neutron magnetic moment. For the neutron, the gyromagnetic ratio $\gamma_{L}$ is negative, which means that the magnetic moment is opposed to the spin angular momentum.

Another interesting property is the effect of a magnetic field applied on the neutron path. A magnetic field exerts a torque on the neutron moment, as it does on any magnetic moment:

$$
\boldsymbol{\Gamma}=\boldsymbol{\mu} \times \boldsymbol{H}=\gamma_{L} \boldsymbol{S} \times \boldsymbol{H} .
$$

As a result of this torque, the magnetic moment of the neutron (and as a consequence the polarization) precesses around the field. Therefore, magnetic fields are perfect tools to control the neutron polarization. In a constant magnetic field, the neutron moment will rotate around the field (Larmor precession) with a frequency:

$$
\omega_{L}=\gamma_{L} H .
$$

A numerical application shows that the rotation is very fast:

$$
\omega_{L}(\mathrm{rad} / \mathrm{sec})=18325 \gamma_{L} H(\text { Gauss })
$$

that is roughly 3000 turns/sec in a 1 gauss field.

Another order of magnitude to have in mind is the precession angle, which is given by:

$$
\frac{\Delta \phi}{\Delta x}(\mathrm{deg} / \mathrm{cm})=2.65 \lambda(\AA) H(\text { gauss }) .
$$

It's easy to understand that the precession angle depends on the wavelength. Indeed, it depends on the time spent in the magnetic field, and therefore on the speed of the neutron. A neutron with wavelength $2.4 \AA$ will precess $60^{\circ}$ flying $10 \mathrm{~cm}$ in a 1 Gauss constant field.

The next important question to consider is what happens if the field varies along the neutron path? Two extreme cases have to be considered. If the field varies slowly compared to the Larmor frequency, the neutron experiences many turns before the field really changes direction. The neutron does not really feel this change and gently follows the field during its rotation. Such a rotation is called "adiabatic". If the field changes abruptly compared to the Larmor frequency from $\boldsymbol{H}_{1}$ to $\boldsymbol{H}_{2}$, the neutron has no time to react: it was rotating around $\boldsymbol{H}_{1}$ and it starts rotating around $\boldsymbol{H}_{2}$. Such a process is called "non adiabatic", and is used to "flip" the neutron polarization, that is to reverse the polarization compared to the guiding fields. Indeed, it is important to realize that flipping a neutron beam is not simply reversing a guide field! In the intermediate cases, when the spatial variation of the field is of the same order of magnitude as the Larmor pulsation, the rotation of the polarization is partial and depends on the adiabaticity parameter $\omega_{L} / \omega_{H}$.

All these properties are at the basis of the production and the manipulation of polarized neutrons. In practice, once the neutron beam has been polarized by a polarizing device, that is a device that transmits neutrons with only one spin state, any magnetic field along the neutron path to the experimental set up (stray field, earth field) is able to rotate the polarization in an uncontrolled way, leading to a partial or even total depolarization of the beam. To prevent such troubles, sufficiently strong guide fields are installed all along the neutron path. If parasitic fields are present, they will only slightly modify the direction of these guide fields, but in the adiabatic limit, so that the total polarization will be preserved. A complete description of the different ways to produce polarized neutrons and of the handling tools used in this technique is out of the scope of this lecture. More details can be found in the lecture book of a previous school devoted to polarized neutron scattering [13].

\section{THE BLUME-MALEYEV EQUATIONS}

Neutrons interact in a sample both with nuclei and magnetic moments. All the different interactions are described in the lecture of M. Enderle. In what follows, we will consider only the two main interactions 


\section{Collection SFN}

as far as strength is concerned: the nuclear one (with the nuclei in the crystal) and the magnetic one (the dipole-dipole interaction with unpaired electrons).

The fundamental equations of polarized neutron scattering have been derived independently by Blume and Maleyev in the beginning of the sixties [7, 8]. When the two periodicities of the magnetic and of the chemical structures are the same, magnetic and nuclear scattering occur at the same points in reciprocal space corresponding to Bragg peaks, and interfere. The differential cross section (proportional to the intensity) of such a mixed reflection is the sum of four terms:

$$
\begin{aligned}
\left(\frac{d \sigma}{d \Omega}\right) & =N^{*} N \\
& +\mathbf{M}_{\perp}^{*} \cdot \mathbf{M}_{\perp} \\
& +\left(N \mathbf{M}_{\perp}{ }^{*}+N^{*} \mathbf{M}_{\perp}\right) \cdot \mathbf{P}_{\mathbf{i}} \\
& +i\left(\mathbf{M}_{\perp}{ }^{*} \times \mathbf{M}_{\perp}\right) \cdot \mathbf{P}_{\mathbf{i}} .
\end{aligned}
$$

In this equation, $\mathbf{P}_{\mathbf{i}}$ is the polarization of the incident beam, $N$ the nuclear structure factor $F_{N}$ and $\mathbf{M}_{\perp}$ the magnetic interaction vector. Due to the dipolar nature of the interaction between the neutron spin and the magnetic moments of unpaired electrons, this latter quantity is not directly the magnetic structure factor $\mathbf{F}_{\mathbf{M}}$ but rather its effective part, that is the projection of $\mathbf{F}_{\mathbf{M}}$ onto the plane perpendicular to the scattering vector $\mathbf{Q}$ :

$$
\mathbf{M}_{\perp}=\left(\mathbf{Q} \times \mathbf{F}_{\mathbf{M}} \times \mathbf{Q}\right)
$$

where both $\mathbf{M}_{\perp}$ and $\mathbf{F}_{\mathbf{M}}$ are complex vector quantities, whereas $N$ is a scalar (also complex in the general case). All these quantities become real in the case of centric structures.

The first term in Eq. (3.1) is a purely nuclear term. The second one is the usual magnetic term that one encounters when treating unpolarized neutron data. Those two terms are independent of the incident polarization. The third term is an interference term between the nuclear and the magnetic signals, whereas the fourth one is the so-called chiral magnetic term. The two latter both depend on the incident polarization.

The direction and the magnitude of the beam polarization can be affected by the scattering process. For the mixed reflection we have considered so far, Blume and Maleyev have also calculated the expression of the scattered (final) polarization $\mathbf{P}_{\mathbf{f}}$ :

$$
\begin{aligned}
\mathbf{P}_{\mathbf{f}}\left(\frac{d \sigma}{d \Omega}\right) & =N^{*} N \mathbf{P}_{\mathbf{i}} \\
& -\left(\mathbf{M}_{\perp}^{*} \cdot \mathbf{M}_{\perp}\right) \mathbf{P}_{\mathbf{i}}+\left(\mathbf{P}_{\mathbf{i}} \cdot \mathbf{M}_{\perp}^{*}\right) \mathbf{M}_{\perp}+\left(\mathbf{P}_{\mathbf{i}} \cdot \mathbf{M}_{\perp}\right) \mathbf{M}_{\perp}^{*} \\
& +\left(N \mathbf{M}_{\perp}^{*}+N^{*} \mathbf{M}_{\perp}\right)-i\left(N \mathbf{M}_{\perp}^{*}-N^{*} \mathbf{M}_{\perp}\right) \times \mathbf{P}_{\mathbf{i}} \\
& -i\left(\mathbf{M}_{\perp}^{*} \times \mathbf{M}_{\perp}\right) .
\end{aligned}
$$

The first nuclear term in Eq. (3.3) does not change the polarization $\left(\mathbf{P}_{\mathbf{f}}=\mathbf{P}_{\mathbf{i}}\right)$. The effect of the non chiral magnetic terms (second line) is a precession of the incident polarization by $180^{\circ}$ around $\mathbf{M}_{\perp}$ : the incident polarization is reversed except the component along $\mathbf{M}_{\perp}$. A careful examination of the interference terms (third line) shows that polarization can be created along $\mathbf{M}_{\perp}$ when magnetic and nuclear signal are in phase. Furthermore, because of the contribution depending on $\mathbf{P}_{\mathbf{i}}$, the polarization rotates on a cone around $\mathbf{M}_{\perp}$. Finally, the chiral magnetic term (fourth line), if present, creates polarization along 
the scattering vector $\mathbf{Q}$. A way to check the existence of such a chiral term is thus to start from an unpolarized beam and measure the final polarization along $\mathbf{Q}$. It is useful at this point to define a convention for further calculations: a right handed set is usually built with the $\mathbf{X}$ axis parallel to the scattering vector, $\mathbf{Y}$ perpendicular to $\mathbf{X}$ in the equatorial plane of the diffractometer and $\mathbf{Z}$ completing the right handed set. With such a convention, the magnetic interaction vector has by definition no component along $\mathbf{X}$, since it is perpendicular to the scattering vector. Before going further, a remark should be made: compared to the equations given just above, some differences, concerning some signs, can be found in the literature. The differences are mainly coming as usual from the different conventions used in quantum mechanics and x-ray crystallography in the definition of plane wave-functions and in the definition of the scattering vectors. To make the story short, those differences play a role only when comes the question to determine an absolute sense of rotation in a chiral structure (left-handed or righthanded). This question has turned out of particular importance in some special cases when comparing neutron polarimetry results to x-ray diffraction data obtained with synchrotron radiation and polarized light and is still debated $[14,15]$.

The relation between the incident and the diffracted polarization is usually summarized in a tensorial form:

$$
\boldsymbol{P}_{f}=\overline{\overline{\mathcal{P}}} \boldsymbol{P}_{i}+\boldsymbol{P}^{\prime}
$$

where $\boldsymbol{P}^{\prime}$ is the created polarization. From Eq. (3.3), one can show that the final polarization $\boldsymbol{P}_{\boldsymbol{f}}$ is either increased or unchanged (in amplitude) by the scattering from a pure state. However, during experiments, one often observe a reduction of the polarization, called depolarization: this depolarization comes from the fact that usually, in real crystals, one is not faced to a pure state, but that different magnetic domains are present. Theses domains play an important role as far as polarized neutrons are concerned and are described in the next section.

\section{MAGNETIC DOMAINS}

Magnetic domains appear when the symmetry of the magnetic structure is less than the symmetry of the paramagnetic phase. If the order of the paramagnetic group is $p$ and the order of the magnetic group is $m$, the number of different domains is $p / m$. Different kind of magnetic domains are encountered in magnetic structures:

- Configuration domains (K domains): configuration domains exist when the propagation vector $\mathbf{K}$ describing the magnetic structure is not transformed either into itself or itself plus a reciprocal lattice vector by all the symmetry operators of the paramagnetic group. The operation of the paramagnetic symmetry on $\mathbf{K}$ generates a set of vectors which is called the star of $\mathbf{K}$. Each distinct vector in the star generates a different configuration domain, and each configuration domain gives rise to a completely separate set of magnetic reflections. These reflections correspond to a single state (when domains of other type are not present). For this reason configuration domains do not give rise to depolarization.

- Orientation domains (S domains): orientation domains occur when the magnetic space group is not congruent with the subgroup that leaves $\mathbf{K}$ invariant. This gives rise to domains in which magnetic moments have directions equivalent with respect to $\mathbf{K}$ and according to the crystal symmetry. These orientation domains contribute to the same reflections in reciprocal space and therefore give rise to depolarization.

- $\mathbf{1 8 0}^{\circ}$ domains: $180^{\circ}$ degree domains are regions of crystal in which the moment directions in one domain are reversed with respect to those in the other. The two domains are related by the time inversion operator. Ferromagnetic domains provide a simple example of this type. When the propagation vector of the magnetic structure is non-zero, these domains cannot be distinguished. They play an important role when the propagation vector is zero, that is when both magnetic and nuclear 


\section{Collection SFN}
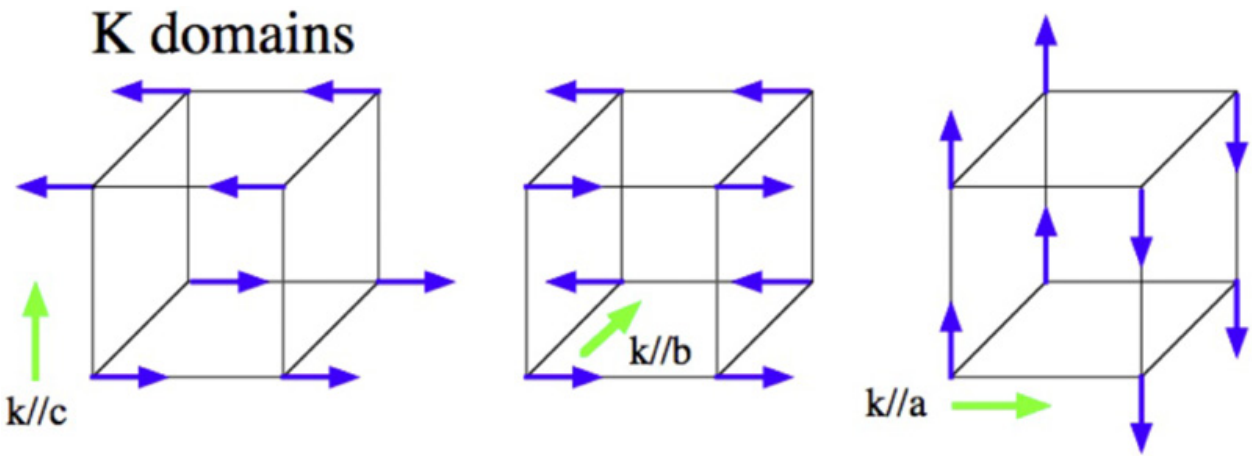

\section{S domains}
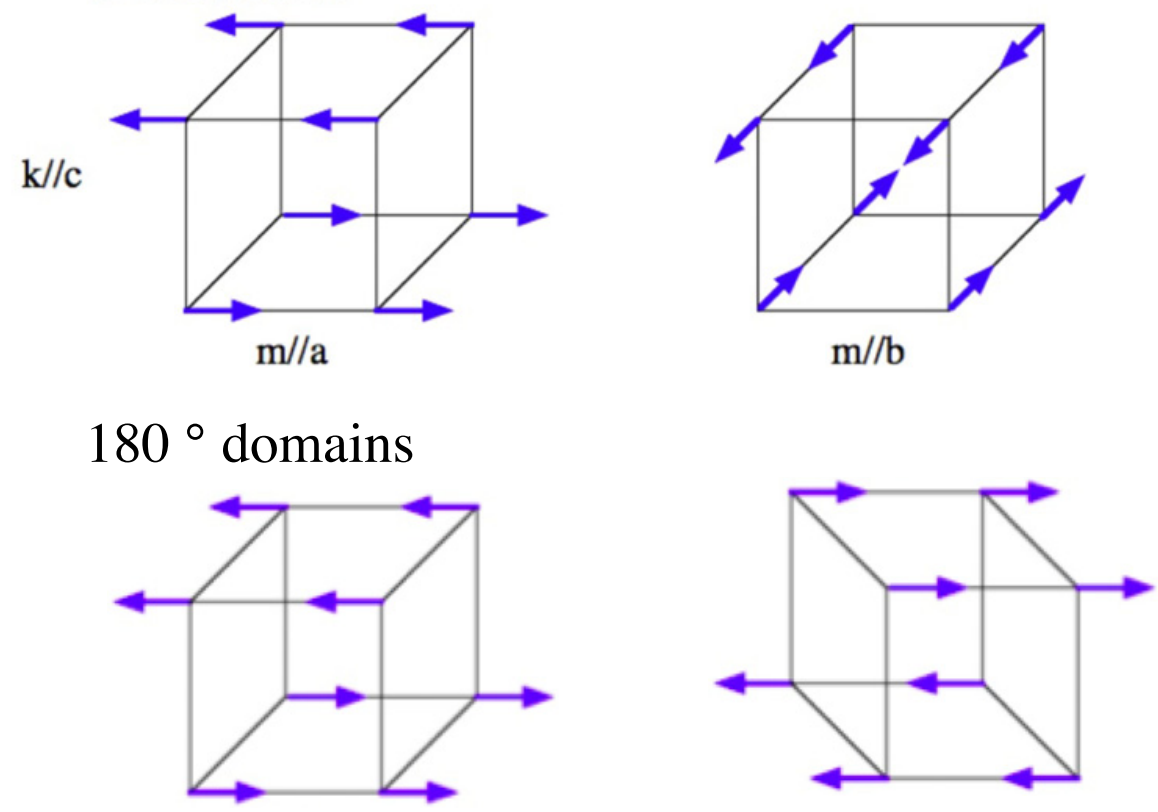

Figure 1. Illustration of the different magnetic domains.

signals occur at the same place in reciprocal space and interfere: the phase difference between the two signals changes by $\pi$ for two such domains.

- Chirality domains: chirality domains occur when the paramagnetic space group is centrosymmetric but the magnetic structure is not. They are related by the space inversion operator. A typical example of chirality domains is encountered in helical magnetic structures, where helices could turn clockwise or anticlockwise.

To illustrate these different points, let us take a simple example, shown in Figure 1, of a primitive cubic space group with a propagation vector $\boldsymbol{K}=(0,0,1 / 2)$. Because of the cubic symmetry, three such $\boldsymbol{K}$ vectors exist and are equivalent: $\boldsymbol{K}_{\mathbf{1}}=(1 / 2,0,0), \boldsymbol{K}_{\mathbf{2}}=(0,1 / 2,0)$ and $\boldsymbol{K}_{\mathbf{3}}=(0,0,1 / 2)\left(\boldsymbol{K}_{\boldsymbol{i}}\right.$ is equivalent to $-\boldsymbol{K}_{\boldsymbol{i}}$ in a primitive space group, so that the total number here is three and not six like it would be in a face centered cubic latice). These three $\boldsymbol{K}$ vectors define the three configuration domains that could be present in the crystal. Let us imagine now that the magnetic moments in the ordered state 
are perpendicular to the propagation vector, directed along the fourfold axis. Within each $\mathrm{K}$ domains, one will find four $\mathrm{S}$ domains, with moments along the (100) and (010) directions (for the $\boldsymbol{K}_{\mathbf{3}}$ domain), these four S domains being two by two $180^{\circ}$ degree domains.

The final polarization scattered from a multidomain sample is obtained from the vector sum of the contributions from the individual domains weighted by their domain fractions. The two Blume-Maleyev equations should be modified according to:

$$
\left(\frac{d \sigma}{d \Omega}\right)=\sum_{j=1}^{n_{d o m}} \alpha_{j}\left(\frac{d \sigma}{d \Omega}\right)_{j}
$$

and

$$
\boldsymbol{P}_{f}\left(\frac{d \sigma}{d \Omega}\right)=\sum_{j=1}^{n_{d o m}} \alpha_{j} \boldsymbol{P}_{f}^{j}\left(\frac{d \sigma}{d \Omega}\right)_{j}
$$

where the sum is taken over the $n_{d o m}$ different domains contributing to the same reflection.

\section{CLASSICAL POLARIZED BEAM TECHNIQUE}

This is the experimental setup used by Nathans and Shull in their pioneering form factor determination in 1959 [9]. In such a simple experiment, the incident neutrons are polarized and the polarization of the scattered beam is not analyzed. A flipping device is used to reverse the incident neutron polarization. The sample is magnetized by a magnetic field applied along the vertical axis $\mathbf{Z}^{\prime}$. Because this field is also used as a guide, the polarization of the neutron beam is also along this axis. The intensities scattered are compared for the two possible states of the incident polarization through their ratio, called the flipping ratio. Such a setup is often referred as "half polarized experiment" nowadays in the literature. For small magnetic amplitudes, the enhancement of sensitivity is tremendous.

Returning to the general equations, one can write the differential cross section (or the intensity) scattered in such an experiment:

$$
I=N^{*} N+\boldsymbol{M}_{\perp}^{*} \cdot \boldsymbol{M}_{\perp}+\boldsymbol{P}_{\boldsymbol{i}} \cdot\left(N \boldsymbol{M}_{\perp}^{*}+N^{*} \boldsymbol{M}_{\perp}\right)+i \boldsymbol{P}_{\boldsymbol{i}} \cdot\left(\boldsymbol{M}_{\perp}^{*} \times \boldsymbol{M}_{\perp}\right) .
$$

If we assume that $\boldsymbol{P}_{\boldsymbol{i}}$ is along the vertical axis $\mathbf{Z}^{\prime}$ (beware that $\mathbf{Z}^{\prime}$ corresponds to $\mathbf{Z}$ we have defined in the previous section only for in-plane reflections, that is when $\alpha$, the angle between the scattering vector and the vertical axis, is equal to $\pi / 2$, see figure below), and that its amplitude is $\pm \mathrm{P}$ when using the flipping device, then:

$$
I^{ \pm}=|N|^{2}+\left|\boldsymbol{M}_{\perp}\right|^{2} \pm P\left[\left(N M_{\perp}^{Z^{\prime} *}+N^{*} M_{\perp}^{Z^{\prime}}\right) \pm i P\left(\boldsymbol{M}_{\perp}^{*} \times \boldsymbol{M}_{\perp}\right)^{Z^{\prime}}\right]
$$

and the flipping ratio, the quantity actually measured is:

$$
R=\frac{I^{+}}{I^{-}}=\frac{|N|^{2}+\left|\boldsymbol{M}_{\perp}\right|^{2}+P\left[\left(N M_{\perp}^{Z^{\prime} *}+N^{*} M_{\perp}^{Z^{\prime}}\right)+i P\left(\boldsymbol{M}_{\perp}^{*} \times \boldsymbol{M}_{\perp}\right)^{Z^{\prime}}\right]}{|N|^{2}+\left|\boldsymbol{M}_{\perp}\right|^{2}-P\left[\left(N M_{\perp}^{Z^{\prime} *}+N^{*} M_{\perp}^{Z^{\prime}}\right)-i P\left(\boldsymbol{M}_{\perp}^{*} \times \boldsymbol{M}_{\perp}\right)^{Z^{\prime}}\right]} .
$$

This equation is general, but rather complex. However, the technique is usually applied in situation for which important simplifications occur. In particular, if the magnetic structure is non chiral (that is when $\boldsymbol{M}_{\perp}$ is parallel to $\boldsymbol{M}_{\perp}^{*}$ ) the last term with the vector product vanishes. If, in addition, the magnetization 


\section{Collection SFN}

is perfectly aligned by the magnetic field $\mathbf{H}$, that is along $\mathbf{Z}^{\prime}$, then $\left|\boldsymbol{M}_{\perp}\right|^{2}, M_{\perp}^{Z^{\prime}}$ and $\mathbf{F}_{\mathbf{M}}$ are simply related via $\alpha$ :

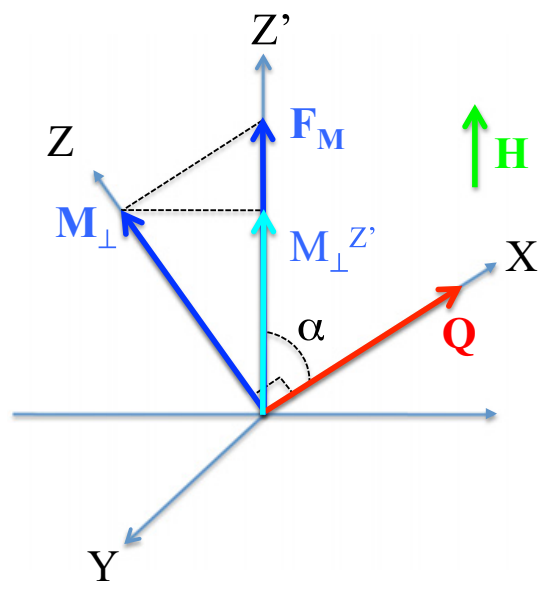

$$
\begin{array}{r}
M_{\perp}=F_{M} \sin \alpha \\
M_{\perp}^{Z^{\prime}}=F_{M} \sin ^{2} \alpha \\
\left|\boldsymbol{M}_{\perp}\right|^{2}=\left|F_{M}\right|^{2} \sin ^{2} \alpha
\end{array}
$$

The expression of the flipping ratio becomes:

$$
R=\frac{N^{2}+\left|F_{M}\right|^{2} \sin ^{2} \alpha+P \sin ^{2} \alpha\left(N F_{M}^{*}+N^{*} F_{M}\right)}{N^{2}+\left|F_{M}\right|^{2} \sin ^{2} \alpha-P \sin ^{2} \alpha\left(N F_{M}^{*}+N^{*} F_{M}\right)}
$$

in which $N$ and $F_{M}$ are scalar quantities, complex in the general case. If, in addition, the structure is centrosymetric, then both the nuclear and the magnetic structure factors are real, and the flipping ratio is further simplified:

$$
R=\frac{N^{2}+F_{M}^{2} \sin ^{2} \alpha+2 P \sin ^{2} \alpha N F_{M}}{N^{2}+F_{M}^{2} \sin ^{2} \alpha-2 P \sin ^{2} \alpha N F_{M}} .
$$

Once the structure is known precisely (that is $N$ ), it is very easy to deduce from the measurement of $R$ the magnetic structure factor $F_{M}$ by solving a second degree equation. This equation has two roots, but the choice between the two roots is in general easy: one of the solution is usually unphysically large. In some cases, the choice is more tricky and one should pay attention.

Another very important remark should be made at this point: this equation, in centric structures, gives not only the amplitude of the magnetic structure factor, but also its phase. The classical polarized beam technique is thus one of the very few technique able to give the phase of a structure factor, phase which is usually lost in a diffraction experiment because what is actually measured is an intensity, that is the square of a structure factor. When the crystal structure is non-centrosymetric, however, the structure factors are complex, and the knowledge of the flipping ratio is not enough to solve the problem. Indeed, one is faced to a single equation for two unknown quantities (the real and the imaginary parts of the magnetic structure factor). It is not possible to extract the magnetic structure factors, and one has to work directly with flipping ratios to treat the data.

Now it is easy to see why the sensitivity is enhanced compared to unpolarized neutrons. Let us imagine that one has to measure a magnetic signal ten times smaller than the nuclear one $\left(F_{M}=\right.$ $0.1 \times N)$. With an unpolarized beam, the intensity of such a mixed reflection is (measured in the equatorial plane, that is when $\alpha=\pi / 2$ ):

$$
I=N^{2}+F_{M}^{2}=N^{2}+(0.1 \times N)^{2}=1.01 \times N^{2} .
$$


The effect of the magnetic contribution is only $1 \%$ of the total signal. If we now turn to polarized neutrons, because of the nuclear/magnetic interference term, the intensities for the two different incoming spin states will be (assuming a perfect polarization of the beam):

$$
\begin{aligned}
& I^{+}=N^{2}+F_{M}^{2}+2 N F_{M}=N^{2}+(0.1 \times N)^{2}+\left(2 \times 0.1 \times N^{2}\right)=1.21 \times N^{2} \\
& I^{-}=N^{2}+F_{M}^{2}-2 N F_{M}=N^{2}+(0.1 \times N)^{2}-\left(2 \times 0.1 \times N^{2}\right)=0.81 \times N^{2} .
\end{aligned}
$$

The effect is now 20\%. The magnetic signal has been amplified by the nuclear one, and therefore the sensitivity is enhanced in a tremendous way. This is why this technique is used for very precise measurement of weak magnetic amplitudes, mainly to determine the form factor of magnetic atoms and magnetization distribution maps within the unit cell. The precision on getting $F_{M}$ from the flipping ratio depends of course on how precisely one is able to know the nuclear structure factor $N$ : this is why the first step of such an investigation is usually an unpolarized neutron experiment at the same temperature. This experiment allows the refinement of the crystal structure, that is the positions of the atoms (including hydrogen atoms when present), the thermal parameters, and the extinction parameters of the crystal. Indeed, extinction affects flipping ratios and should be corrected when calculating magnetic structure factors.

Looking at expression (5.8), it is also easy to understand why $180^{\circ}$ degree domains play an important role with polarized neutrons, as soon as the interference term is concerned. Indeed, for the two $180^{\circ}$ degree domains, the nuclear structure factor $N$ is the same, whereas the magnetic structure factor $F_{M}$ changes sign. The interference term will therefore change sign from one domain to the other, and if the two domains have the same volume, the superposition of the two signals will even result in a cancellation of this interference term, leading to a flipping ratio equal to one. This is at the origin of the limitations on the class of compounds that can be investigated by this technique: paramagnets under an applied field (to induce a long range magnetization), saturated ferromagnets (to have a single domain), or saturated ferrimagnets. For antiferromagnets, a large attention should be paid: only a very few particular cases, with several Bravais sublattices and in-phase nuclear and magnetic structure factors can be investigated, under the condition that one is able to unbalance $180^{\circ}$ degree domains. In most of the cases with antiferromagnets, the technique gives access only to the ferromagnetic component induced by the applied magnetic field, and not to the antiferromagnetic one that cancels out because of domains. Last but not least, since the technique relies on the interference term, magnetic and nuclear signals should appear at the same position in reciprocal space, which means that only magnetic structures with a $\mathbf{K}=\mathbf{0}$ propagation vector can be investigated.

Anyway, when performing such an experiment and treating the data, one should always remember what are the underlying hypothesis that have been used from the very beginning to simplify the problem: absence of any chiral magnetic term on the one hand, and magnetization aligned with the applied magnetic field in the vertical direction on the other hand. If this is not the case, the usual treatment, that is the extraction of the magnetic structure factors from the flipping ratios, would lead to erroneous results. In such cases, one should go back to the general Eq. (5.3) and treat data with this formula.

Indeed, how to treat data? In centric cases, the experiment gives the magnetic structure factors $F_{M}$ of the different Bragg reflections $\mathbf{Q}$. These $F_{M}$ 's are the Fourier components of the total magnetization distribution $\rho(\mathbf{r})$ (a distribution in direct space), which can also be defined as the product of the magnetic moment $\boldsymbol{m}_{v}$ of atom $v$ and its form factor $f_{v}(\boldsymbol{Q})$ (defined in the reciprocal space):

$$
\boldsymbol{F}_{\boldsymbol{M}}(\boldsymbol{Q})=\sum_{v} f_{v}(\boldsymbol{Q}) \boldsymbol{m}_{v} e^{i \boldsymbol{Q} \cdot \boldsymbol{r}_{v}}=\int_{\text {cell }} \boldsymbol{\rho}(\mathbf{r}) e^{i \mathbf{Q} \cdot \mathbf{r}} d \mathbf{r} .
$$

In the case of spin-only ions, the form factor really represents the Fourier transform of the electronic distribution of unpaired electrons. However, in the presence of an orbital contribution, the magnetization distribution is not simply related to the electronic distribution, and both a spin and an orbital form 


\section{Collection SFN}

factor should be defined. In that case, accurate calculations are rather tedious, but if they are done correctly, comparison with the experiment gives important information about the ground state of unpaired electrons in the solid.

Retrieving the shape of the distribution $\boldsymbol{\rho}(\mathbf{r})$ in real space from the measured Fourier coefficients $F_{M}$ is a typical inverse Fourier problem. Several approaches exist [16], and belong to two main families: direct (model free) methods and parametrized models.

\subsection{Direct methods}

Direct methods use nothing but the experimental data, and are therefore a necessary step before any attempt to refine a model. Since the experiment gives the Fourier components of the magnetization distribution, the first idea to retrieve this distribution in real space is to use the Fourier inversion ( $\mathrm{V}$ is the volume of the unit cell):

$$
\rho(\boldsymbol{r})=\frac{1}{V} \sum_{\boldsymbol{Q}} F_{M}(\boldsymbol{Q}) e^{-i \boldsymbol{Q} \cdot \boldsymbol{r}} .
$$

This technique is very simple to operate and has been widely used in the past. However, the number of measured reflections is necessarily limited and, with this method, all the coefficients which have not been measured are considered as being equal to zero. This default, combined to the fact that no use is made of the standard deviations of the observations, results in spurious spatial correlations and affects the quality of the reconstructions. In addition, this technique can only be applied to magnetic structure factors, and is therefore restricted to centric cases.

A major breakthrough in the way of treating polarized neutron diffraction data has been reached with the Maximum of Entropy solution (Maxent). This method, based on Bayesian statistical techniques, was first applied to radio astronomy [17] and has been transposed to magnetization reconstructions by Papoular [18]. Without entering the details, it essentially provides the least informative map consistent with the observations, taking into account their standard deviations. The considerable advantage of this method relies on the fact that no assumptions are made about non-observed Fourier coefficients. The quality of the reconstructions is incomparably improved compared to the classical Fourier inversion.

A nice illustration of this improvement is shown in Figure 2, where the projections of the magnetization distribution obtained by Fourier inversion and by the Maxent technique are compared in $\mathrm{MnO}$ [19], and in the high $\mathrm{T}_{c}$ superconductor $\mathrm{YBa}_{2} \mathrm{Cu}_{3} \mathrm{O}_{7}$ [20]. In the case of $\mathrm{MnO}$, the complete data set (cubic space group of high symmetry, measured at short wavelength on a hot-source, with a relatively strong induced signal) allows, even simply with a raw Fourier transform, to obtain a very good reconstruction of the magnetization distribution in direct space. Maxent only cleans some small oscillations visible between the main peaks of magnetization carried by the manganese atoms. In the case of $\mathrm{YBa}_{2} \mathrm{Cu}_{3} \mathrm{O}_{7}$, the result is more spectacular: this data set was much more reduced, because of a longer wavelength and a lower crystal symmetry. In addition, the signal was pretty weak. As a result, the raw Fourier transform is rather bad: no clear signal is visible. All the artifacts present in the Fourier maps which are mainly due to truncation effects are suppressed in the Maxent reconstruction, which reveals the main contributions on the copper atoms but also a small signal on the bridging oxygens.

Last but not least, another advantage of the Maxent technique arises from the fact that it has been extended to acentric structures [21], and can thus operate directly from the experimental flipping ratios.

\subsection{Parametrized models}

Once the system is well enough understood for a model to be proposed, several procedure can be carried out, corresponding to different level of approximations. Two different approaches are generally used in model refinements. The first one, the multipolar expansion technique, was originally developed for 
(a)

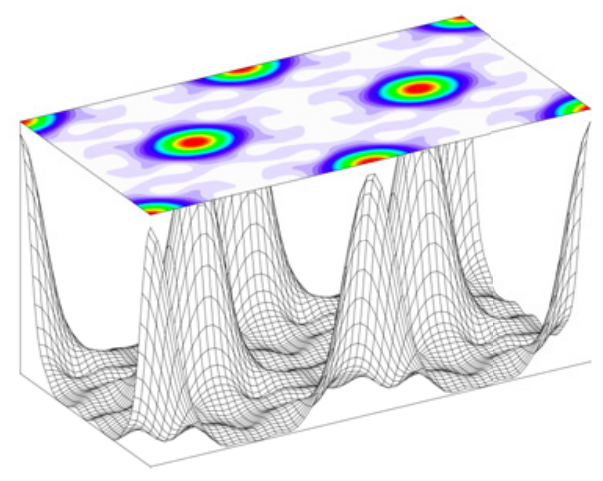

(c)

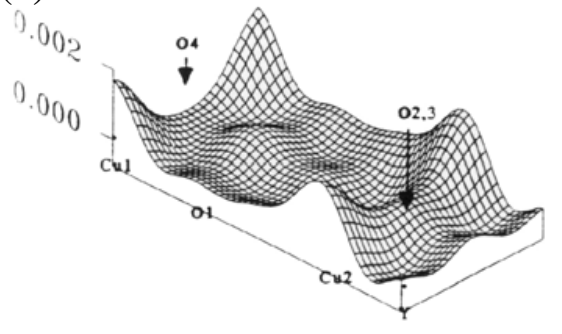

(b)
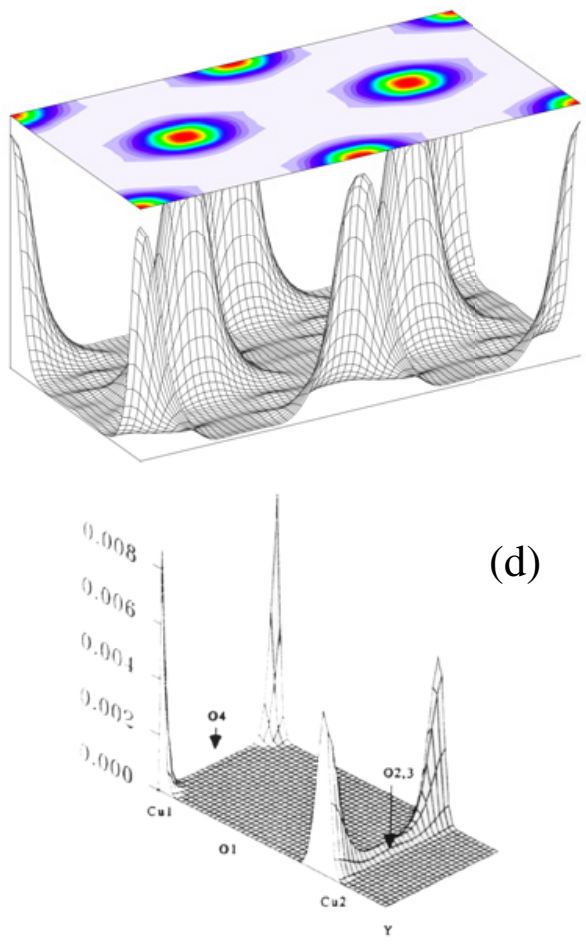

Figure 2. Magnetization distribution in $\mathrm{MnO}$ obtained by Fourier inversion (a) and by the Maxent technique (b). Magnetization distribution in the high $\mathrm{T}_{c}$ superconductor $\mathrm{YBa}_{2} \mathrm{Cu}_{3} \mathrm{O}_{7}$ by Fourier inversion (c) and by Maxent (d). From Refs. [19] and [20].

electronic densities derived from x-ray measurements [22] and is the more flexible. The magnetization distribution $\rho(\mathbf{r})$ in direct space is expanded onto a basis set of real spherical harmonic functions centered at the atomic positions according to:

$$
\rho(\mathbf{r})=\sum_{i}^{\text {atoms }} \rho_{i}^{\text {mult }}\left(\mathbf{r}_{\mathbf{i}}\right)
$$

and

$$
\rho_{i}^{\text {mult }}\left(\mathbf{r}_{\mathbf{i}}\right)=\sum_{l=0}^{l_{\max }=4} R_{l}^{i}\left(\kappa r_{i}\right) \sum_{m=-l}^{l} P_{l m}^{i} y_{l m}^{i}(\theta, \varphi)
$$

in which $y_{l m}^{i}(\theta, \varphi)$ are real spherical harmonic functions and $P_{l m}^{i}$ their populations. The Slater-type radial wavefunction $R_{l}^{i}(\kappa r)$ are expressed as:

$$
R_{l}^{i}(\kappa r)=\frac{\kappa^{3} \zeta_{l}^{n_{l}+3}}{\left(n_{l}+2\right) !} r_{i}^{n_{l}} e^{-\kappa \zeta_{l}^{i} r_{i}}
$$

where $\kappa$ is a contraction coefficient that can be refined and $\zeta$ is twice the standard Slater exponent tabulated for the atoms (as the magnetization density corresponds to a square of atomic orbital). $n_{l}$ is an integer that depends on the nature of the orbital (s, p, d ...). The magnetic structure factor can then be 


\section{Collection SFN}
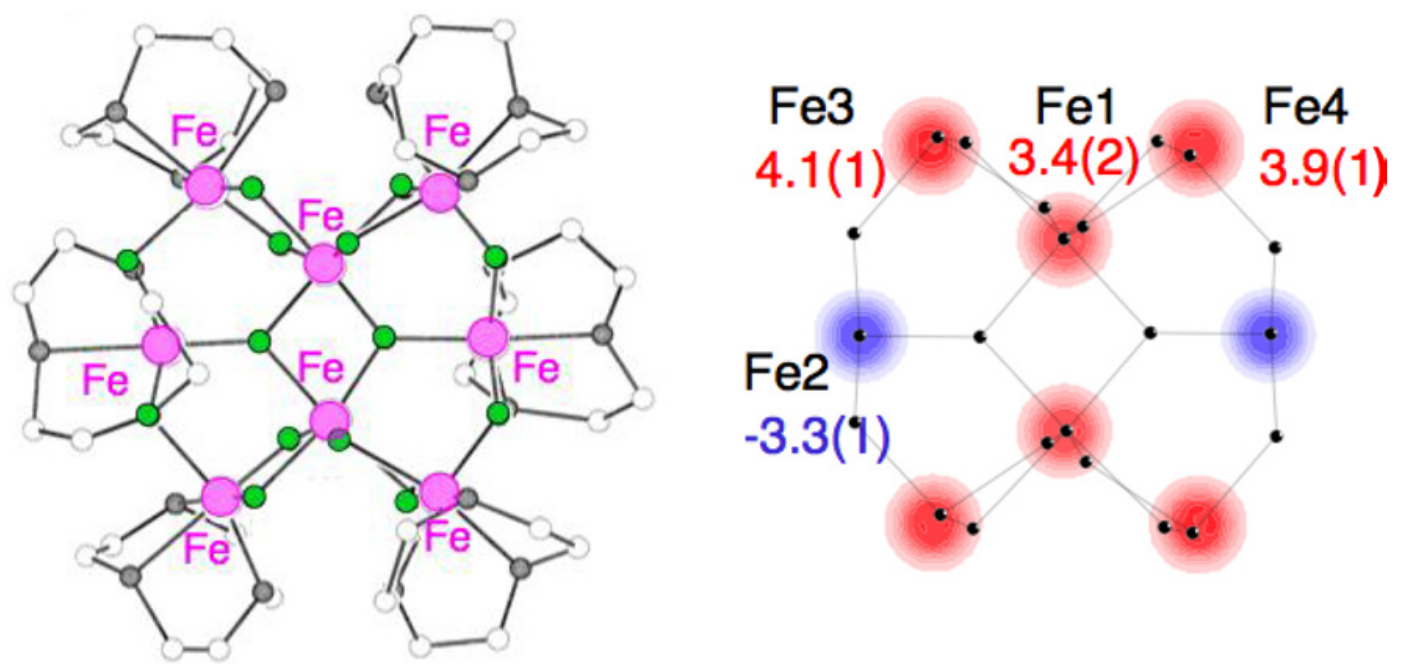

Figure 3. View of the $\mathrm{Fe}_{8}$ molecular cluster (left) and experimental magnetization distribution (right) as measured by polarized neutron diffraction. From Ref. [23].

derived by Fourier transform of $\rho(\mathbf{r})$ :

$$
F_{M}(\mathbf{Q})=\sum_{i}^{\text {atoms }} \sum_{l=0}^{4} i^{l}\left\langle j_{l}(\mathbf{Q})\right\rangle \sum_{m=-l}^{l} P_{l m} y_{l m}\left(\theta_{Q}, \varphi_{Q}\right) e^{i \mathbf{Q} \cdot \mathbf{R}_{\mathbf{i}}} e^{-W_{i}} .
$$

The parameters to be refined are the populations $P_{\ell}^{m}$ (amplitudes of the harmonic functions) and the radial exponents $\kappa$. In acentric cases, the refinement can be carried out directly on flipping ratios.

Alternatively, in the so-called wave function approach, a Hartree-Fock type magnetic wave function $|\Psi\rangle$ is constructed from standard atomic orbitals at each magnetic site:

$$
|\Psi\rangle=\sum_{i}^{\text {atoms }} \alpha_{i} \psi_{i}(\mathbf{r})
$$

in which the $\psi_{i}(\mathbf{r})$ 's are atomic orbitals. The parameters of the model are the coefficients $\alpha_{i}$. In the pure spin case (when there is no orbital contribution), the magnetic structure factor can be expressed as:

$$
F_{M}(\mathbf{Q})=\left\langle\Psi\left|e^{i \mathbf{Q} \cdot \mathbf{r}}\right| \Psi\right\rangle=\int \Psi^{*} e^{i \mathbf{Q} \cdot \mathbf{r}} \Psi d \mathbf{r}
$$

Two types of terms enter the expression (5.19), namely one center integrals (orbitals on the same atomic site), which represent the main contributions, and two center integrals, which are correcting terms corresponding to the overlap between the wave functions of neighboring atoms. These latter integrals are rather tedious to evaluate, and usually, an approximation consists in restricting the calculations to the one center integrals only, that is to consider the magnetization as the sum of magnetization due to individual atoms. Here again, refinements can be carried out directly on flipping ratios.

Such treatments (parametrized models) have been widely used for magnetic molecular compounds either with the wave function approach or with the more flexible multipolar expansion. A typical example is raised with the well-known molecule $\mathrm{Fe}_{8}$ (Fig. 3) [23]. This cluster has attracted special attention in recent years as displaying pure quantum tunneling of the magnetization. Another interesting feature is the strong magnetic anisotropy of the cluster, although each $\mathrm{Fe}^{I I}$ ion $(\mathrm{S}=5 / 2)$ is individually isotropic. The structure of the cluster consists in eight Fe ions linked by bridges. A model with six 


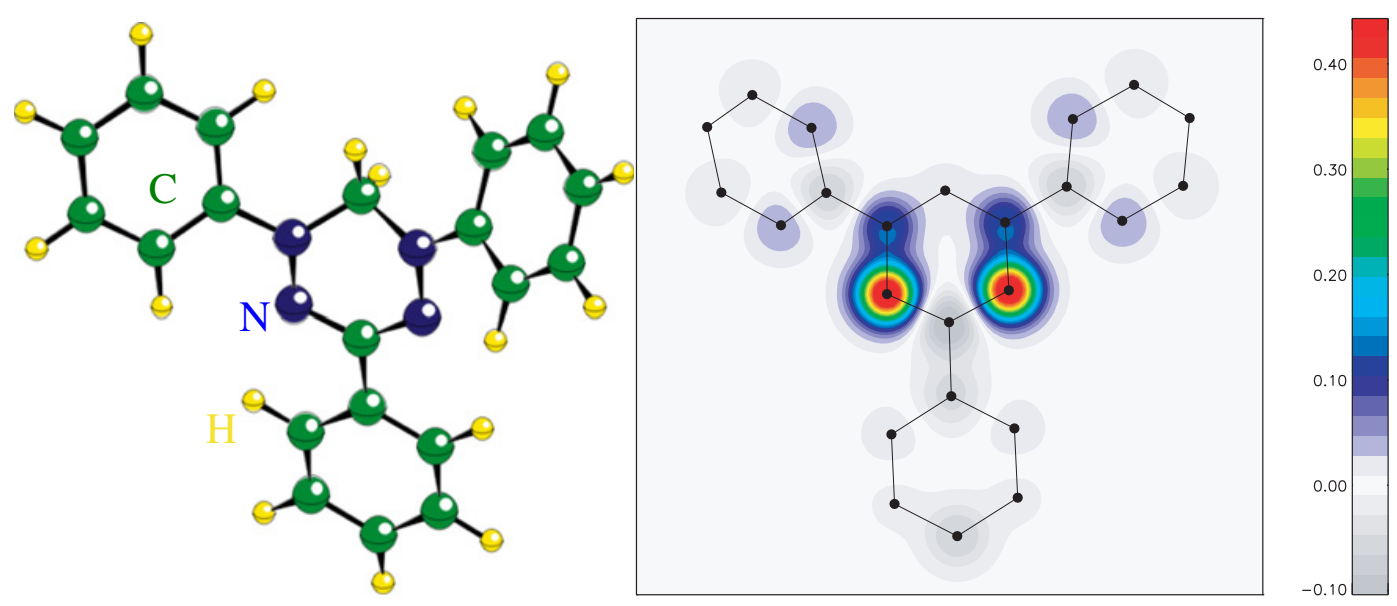

Figure 4. View of the TPV molecule (left) and experimental magnetization distribution (right) as measured by polarized neutron diffraction.

spin up and two spin down iron ions was proposed to describe the spin configuration of the magnetic ground state $S=10$, resulting from the competition between various intra-cluster antiferromagnetic interactions. In order to determine without any ambiguity the distribution of the spins in the ground state, the magnetization distribution was measured by polarized neutron diffraction. This experiment confirmed that six ions (the two body ions of the butterfly configuration and the four peripheral ions) carry spins parallel to the applied field (in red), while the ions of the middle of the wings carry opposite spins (in blue in Fig. 3) .

Another example is given on Figure 4. It concerns the magnetization distribution in the free radical triphenylverdazyl (TPV). This organic compound is a stable free radical with one unpaired electron (with spin 1/2), built from carbon (green), nitrogen (blue) and hydrogen (yellow) atoms. The main question in such a molecule is to know where is (de)localized the spin $1 / 2$ ? Indeed, in conventional insulating magnetic materials, the magnetization distribution is generally the sum of contributions due to individual atoms, each of them being characteristic of a particular configuration of the free atom or ion. The degeneracy of the different orbital states is removed by the crystal field. As a result, the magnetic moments are well localized around the atoms, and the shape of the distribution corresponds to one particular atomic orbital. In ionic compounds made of magnetic cations and diamagnetic anions, a small part of the density on the metal can be transferred onto the ligands, but this fraction is generally weak and does not strongly affect the nature of the magnetic orbital on the metal. In the case of free radicals, the situation is strongly different. In the framework of elementary molecular orbital theory, the magnetism of a single radical is attributed to an unpaired electron residing on the singly occupied molecular orbital (SOMO). This molecular orbital is built up from the individual atomic orbitals of the atoms constituting the molecule. It can happen that different orbitals on different atomic sites strongly contribute to this SOMO, resulting in one unpaired electron being strongly delocalized over a complete molecule. The spin is no longer well localized on one particular atom, but is spread over a molecule or a part of the molecule. This property is often referred as "spin delocalization" in the literature. It is the case for TPV: the unpaired electron is delocalized over the complete molecule and the largest part of the magnetization is carried by the two nitrogen atoms which are not connected to phenyl rings. The nitrogen atoms connected to phenyl rings carry three times less magnetization. In addition, several carbon atoms carry negative magnetization (in grey), that is opposed to the applied magnetic field. In molecular orbital theory, the unpaired electron of the radical occupies the SOMO. This orbital can be 


\section{Collection SFN}

delocalized on a part of the molecule, resulting in a magnetization also spread out (spin delocalization effect). However, at the restricted Hartree-Fock level, where all the doubly occupied molecular orbitals have a unique energy, this density is positive (or zero) everywhere. This view is actually oversimplified. The magnetization on certain atoms contributing to the SOMO can be negative, that is in opposition to the total magnetization. To account for this effect, called "spin polarization effect", one has to go beyond the restricted Hartree-Fock scheme. A complete description of this effect can be found in Ref. [29]. The alternating signs on the phenyl rings is of the same origin.

Both the multipolar expansion and the wave function approach we just described are examples of modelisation of the magnetization distribution in direct space, and are particularly well adapted to molecules. For isolated magnetic ions, rigorous calculations of the form factor can be performed. The general treatment including an orbital contribution can be found in Marshall and Lovesey [24], Lovesey [25], Lander and Brun [26], Stassis and Deckman [27] and is developed in the chapter of Ballou in the lecture book of a preceding Polarized Neutron Diffraction school [13]. Without entering the rather complicated mathematical details, it can be shown that the magnetic structure factor can be expressed on each magnetic atom as the product of the total magnetic moment (spin + orbit) and of the form factor according to the expression (5.12). The form factor on each site takes the form:

$$
f(Q)=\sum_{L} C_{L}\left\langle j_{L}(Q)\right\rangle
$$

where the $\left\langle j_{L}(Q)\right\rangle$ 's are the radial integrals (Bessel-Fourier transform of order L) of the radial density. They depend on the element, on the shell, and on the valency. They have been calculated for transition metals, rare earth and actinides in various states and tabulated by Brown [28]. The $C_{L}$ coefficients depend on the relative occupancies of the orbitals, on the orbital contributions, and on the direction of $\mathbf{Q}$ with respect to these orbitals. $L$ is connected to $\ell$, quantum number of the shell under consideration, through triangular relations $(L \leq 2 \ell)$.

In the case of transition metal, rare earth and actinides, a further approximation is often encountered, and known as "dipolar approximation". This approximation is the simplest one that considers ions as having a spherical symmetry. Then only $C_{0}$ and $C_{2}$ are non-zero in (5.20) which becomes:

$$
f(Q)=\left\langle j_{0}(Q)\right\rangle+C_{2}\left\langle j_{2}(Q)\right\rangle
$$

$C_{2}$ gives the ratio between the orbital and the total moment:

$$
C_{2}=\mu^{\text {orbit }} /\left(\mu^{\text {spin }}+\mu^{\text {orbit }}\right) .
$$

This approximation is very often used. In particular, this is the underlying approximation in all the programs refining magnetic structures from integrated intensities. Figure 5 shows the magnetization distributions in direct space and the corresponding form factors in reciprocal space for $\mathrm{MnO}$ [19] and $\mathrm{CoO}$ [30]. In the case of $\mathrm{MnO}$, the magnetic ion is completely isotropic, as expected for a $\mathrm{S}=5 / 2$ spin state. There is no orbital contribution and the repartition $e g / t 2 g$ is as expected, three electrons in the $t 2 g$ orbitals and two in the eg ones. One can clearly see on the form factor that all the observed experimental points are falling on the dipolar fit curve. On the contrary, for $\mathrm{CoO}$, Maxent already reveals an anisotropy of the distribution around the Co atoms due to the $e g / t 2 g$ repartition. It also evidenced a signal on the oxygen atoms (between the Co ions). The form factor refinement confirms these two features and also points out an orbital contribution. As a result, one can clearly see that experimental points are no longer falling on the dipolar curve, but are scattered on each side of this curve.

To finish with the classical polarized beam technique, we have seen that one of the underlying hypothesis is that magnetization is aligned with the applied magnetic field $\mathbf{H}$. In the case of very anisotropic compounds this is not true. To overcome this problem, Gukasov and Brown [31] have proposed to use atomic site susceptibility tensor $\chi_{i j}$ (see Fig. 6) and write that on each site the induced 
$\mathrm{MnO}$
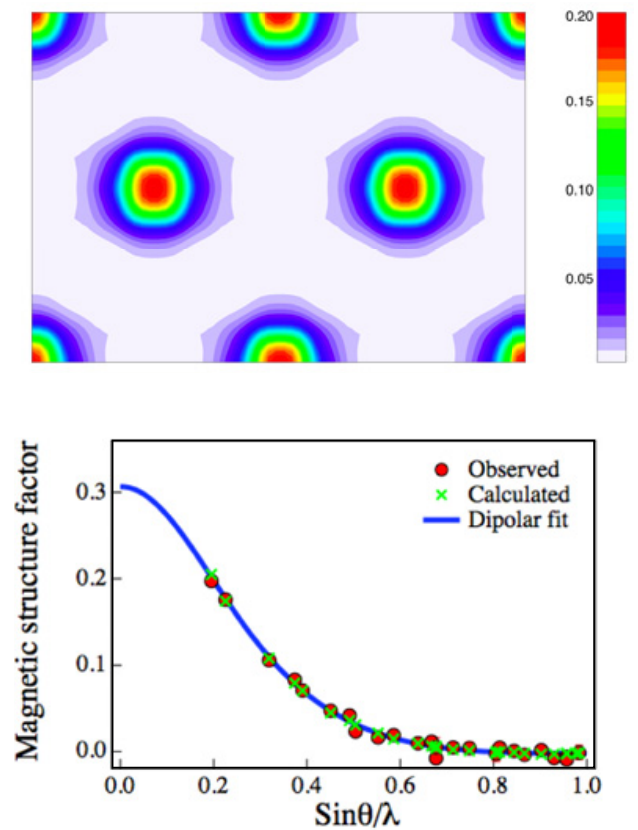

$\mathrm{CoO}$
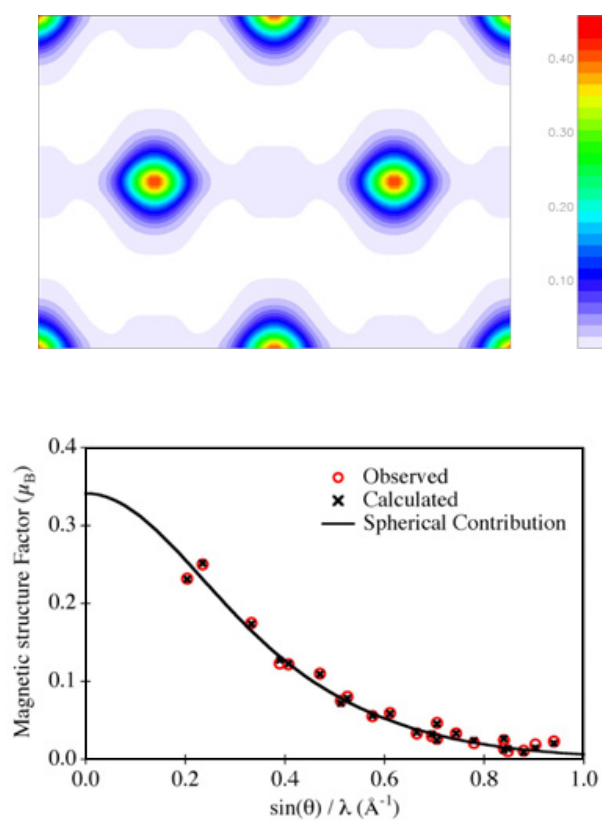

Figure 5. Magnetization distribution in direct space (up) and form factor in reciprocal space (bottom) for $\mathrm{MnO}$ (left) and $\mathrm{CoO}$ (right). From Refs. [19] and [30].

$$
\text { CUBIC, } \chi_{11}=\chi_{22}=\chi_{33}
$$

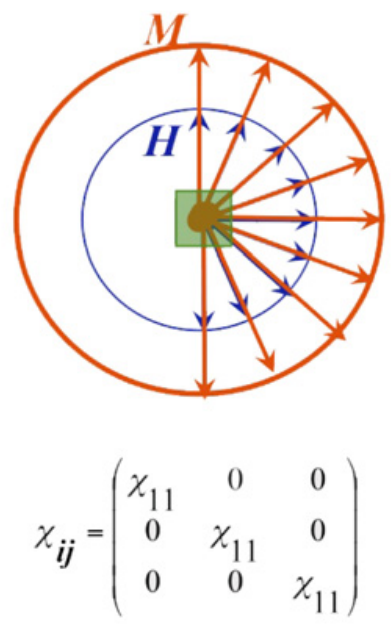

UNIAXIAL $\chi_{11}=\chi_{22}<\chi_{33}$

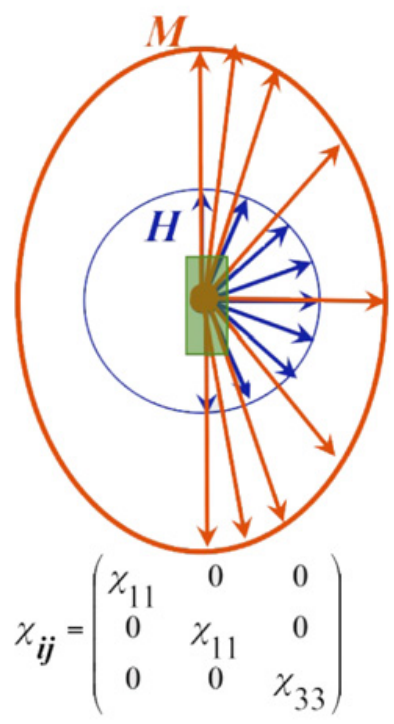

Figure 6. Atomic site susceptibility tensor. 


\section{Collection SFN}

magnetic moment is:

$$
M_{i}=\sum_{j} \chi_{i j} H_{j} .
$$

Calculations are then carried out using the general Eq. (5.3).

To conclude this section, form factor studies can bring very accurate information related to the spatial distribution of unpaired electrons in magnetic compounds. The price to pay is a lot of effort in performaing accurate measurements and in a careful analysis of the data.

\section{LONGITUDINAL POLARIZATION ANALYSIS (LPA)}

In 1969 Moon, Riste and Koehler have shown that it was possible to increase the power of a polarized neutron spectrometer by adding a polarization analyzer after the sample [10]. They managed to operate the first triple axis machine with both incident polarized neutrons and polarization analysis capabilities, giving access to many new pieces of information, as we will see. The main characteristic of such experiments is that if the neutrons are polarized in a particular direction of space, the analysis is done in the same direction. This is why the method is called "uniaxial" or "longitudinal". This property comes from the fact that such experiments are performed with relatively strong magnetic guide fields applied around the sample to preserve the incident polarization. Because of the precession of the polarization vector around magnetic fields that we have discussed in the first sections, only the component along the guide field (the "longitudinal" one) is preserved and can be analyzed, the transverse components being lost.

Combining the two possible states of the incident polarization and the two possible states for the scattered one, it is possible to measure four different intensities: $I^{++}, I^{+-}, I^{-+}$and $I^{--}$. Going back to Blume's equation, one can see in Eq. (3.3) terms proportional to $\mathbf{P}_{\mathbf{i}}$ (which do not change the incident polarization) and terms proportional to $-\mathbf{P}_{\mathbf{i}}$ (which reverse completely the incident polarization). Following the usual terminology, the former will be called non spin-flip (NSF) terms and the latter spin-flip (SF) terms. A very general rule is that a spin flip process is of magnetic origin. For non spin flip process, it is more subtle: it can be either nuclear or magnetic. Indeed, magnetic components parallel to $\mathbf{P}_{\mathbf{i}}$ are NSF, whereas magnetic components perpendicular to $\mathbf{P}_{\mathbf{i}}$ are $\mathrm{SF}$.

$\mathbf{P}_{\mathbf{i}}$ can be chosen along any direction $\mathbf{X}, \mathbf{Y}$ or $\mathbf{Z}$ (defined from the beginning as along $\mathbf{Q}$, perpendicular to it in the scattering plane, and vertical when $\mathbf{Q}$ is confined in the equatorial plane). Combining these three directions to the four possible combinations of neutron spin, one hand up with twelve different cross sections, six spin-flip and six non spin-flip. Defining $M_{c h}=i\left(M_{\perp}^{Z *} M_{\perp}^{Y}-\right.$ $\left.M_{\perp}^{Y *} M_{\perp}^{Z}\right), R_{Y}=N M_{\perp}^{Y *}+N^{*} M_{\perp}^{Y}$ and $R_{Z}=N M_{\perp}^{Z *}+N^{*} M_{\perp}^{Z}$, one can write (P being the value of the incident beam polarization):

$$
\begin{gathered}
I_{X}^{++} \propto|N|^{2} \\
I_{X}^{--} \propto|N|^{2} \\
I_{X}^{+-} \propto\left|M_{\perp}\right|^{2}-P M_{c h} \\
I_{X}^{-+} \propto\left|M_{\perp}\right|^{2}+P M_{c h} \\
I_{Y}^{++} \propto|N|^{2}+\left|M_{\perp}^{Y}\right|^{2}+P R_{Y} \\
I_{Y}^{--} \propto|N|^{2}+\left|M_{\perp}^{Y}\right|^{2}-P R_{Y} \\
I_{Y}^{+-} \propto\left|M_{\perp}^{Z}\right|^{2}
\end{gathered}
$$




$$
\begin{gathered}
I_{Y}^{-+} \propto\left|M_{\perp}^{Z}\right|^{2} \\
I_{Z}^{++} \propto|N|^{2}+\left|M_{\perp}^{Z}\right|^{2}+P R_{Z} \\
I_{Z}^{--} \propto|N|^{2}+\left|M_{\perp}^{Z}\right|^{2}-P R_{Z} \\
I_{Z}^{+-} \propto\left|M_{\perp}^{Y}\right|^{2} \\
I_{Z}^{-+} \propto\left|M_{\perp}^{Y}\right|^{2} .
\end{gathered}
$$

By measuring the SF or NSF cross sections for the three orientations of the incident polarization, $\mathbf{P}_{\mathbf{i}} / / \mathbf{X}$, $\mathbf{P}_{\mathbf{i}} / / \mathbf{Y}$ and $\mathbf{P}_{\mathbf{i}} / / \mathbf{Z}$, it is possible to have access independently to all the different terms. This method is known as the (generalized) vertical field-horizontal field (VH-VF) method or XYZ method.

$$
\begin{gathered}
|N|^{2} \propto I_{X}^{++}=I_{X}^{--} \\
\left|M_{\perp}^{Y}\right|^{2} \propto \frac{I_{Y}^{++}+I_{Y}^{--}}{2}-\frac{I_{X}^{++}+I_{X}^{--}}{2}=\frac{I_{X}^{+-}+I_{X}^{-+}}{2}-\frac{I_{Y}^{+-}+I_{Y}^{-+}}{2} \\
\left|M_{\perp}^{Z}\right|^{2} \propto \frac{I_{Z}^{++}+I_{Z}^{--}}{2}-\frac{I_{X}^{++}+I_{X}^{--}}{2}=\frac{I_{X}^{+-}+I_{X}^{-+}}{2}-\frac{I_{Z}^{+-}+I_{Z}^{-+}}{2} \\
M_{c h} \propto \frac{I_{X}^{-+}-I_{X}^{+-}}{2} \\
R_{Y} \propto \frac{I_{Y}^{++}-I_{Y}^{--}}{2 P} \\
R_{Z} \propto \frac{I_{Z}^{++}-I_{Z}^{--}}{2}
\end{gathered}
$$

$M_{c h}, R_{Y}$ and $R_{Z}$ can also be determined by measuring the polarization creation respectively in the direction $\mathbf{X}, \mathbf{Y}$ or $\mathbf{Z}$, starting from an initially unpolarized beam $\left(\mathbf{P}_{\mathbf{i}}=\mathbf{0}\right)$ :

$$
\begin{aligned}
& P_{f}^{X} \propto \frac{M_{c h}}{I_{0}} \\
& P_{f}^{Y} \propto \frac{R_{Y}}{I_{0}} \\
& P_{f}^{Z} \propto \frac{R_{Z}}{I_{0}}
\end{aligned}
$$

in which $I_{0}=|N|^{2}+\left|M_{\perp}\right|^{2}$ is related to the total unpolarized intensity.

The most interesting situation is realized when $\mathbf{P}_{\mathbf{i}} \| \mathbf{Q}$ (along $\mathbf{X}$ ), because in that case the magnetic contributions are purely SF and the structural ones purely NSF. In other words, there is a complete separation of nuclear and electronic contributions. By simply scanning a reflection in the two SF and NSF channels in this configuration immediately tells you the nature of this reflection: purely nuclear, purely magnetic or mixed nuclear/magnetic.

A very interesting example of application of LPA is met with the multiferroic compound $\mathrm{Ni}_{3} \mathrm{~V}_{2} \mathrm{O}_{8}$ (see Fig. 7). The ferroelectricity in this compound is of magnetic origin and emerges from a cycloidal spin order. The question was to know whether the spin helicity, that is the sense of rotation of the spins within the cycloids (clockwise or counterclockwise), was connected to the direction of the spontaneous electric polarization, and if it was possible to control this sense by an electric field applied on cooling the sample [32]. The authors have investigated this helicity by LPA, in a geometry where $\mathbf{P}_{\mathbf{i}} / / \mathbf{Q}$. Figure $7 \mathrm{c}$ shows Q-scan profiles of a magnetic satellite. There is a strong asymmetry in the intensity between the $(+-)$ and $(-+)$ configurations which reverses with the direction of the applied electric field $\mathbf{E}$. It can be 


\section{Collection SFN}

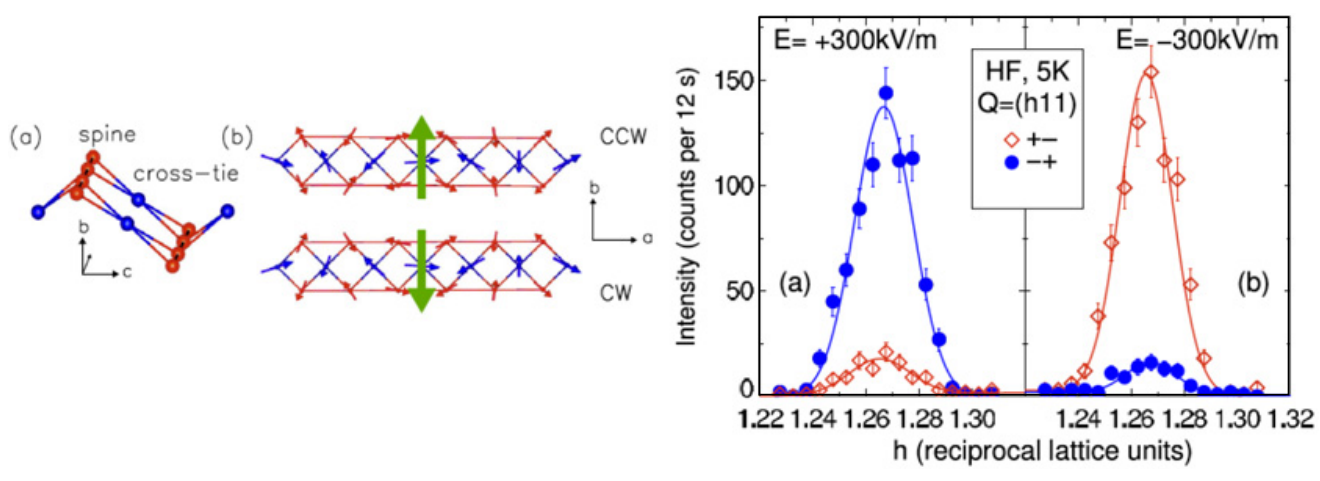

Figure 7. Left: (a) $\mathrm{Ni}_{3} \mathrm{~V}_{2} \mathrm{O}_{8}$ crystal sublattice showing $\mathrm{Ni}^{2+}$ spine (red) and cross-tie (blue) sites. (b) Counterclockwise (top) and clockwise (bottom) spin cycloids propagating along the a axis. The (green) vertical arrow indicates the direction of $\mathrm{P}$ (electric polarization). Right: full polarized magnetic diffraction in an electric field under opposite spin-flip scattering conditions. (a) Cooling in a field $\mathrm{E}=+300 \mathrm{kV} / \mathrm{m}$ from 11 to $5 \mathrm{~K}$ (E-cooling) favors cycloidal domains that predominantly diffract neutrons polarized antiparallel to Q. (b) Reversing E yields the opposite polarized intensity asymmetry. From Ref. [32].

shown that each of these two configurations $(+-)$ and $(-+)$ is more sensitive to one of the two magnetic domains characterized by a different helicity. This experiment thus demonstrates in a qualitative fashion the E-driven suppression and promotion of cycloidal magnetic domains.

In fact, as far as the question of handedness is concerned, even a simpler experimental setup is sufficient to address the question. This was the case for the study of another multiferroic compound, $\mathrm{TbMnO}_{3}$ (see Fig. 8). The ferroelectricity in this compound is also of magnetic origin and emerges from a cycloidal spin order, just as in the case of $\mathrm{Ni}_{3} \mathrm{~V}_{2} \mathrm{O}_{8}$. The same questions, that is to know whether the spin helicity was connected to the direction of the spontaneous polarization, and if it was possible to control this sense by an electric field was answered by a slightly different polarized neutron experiment [33]. The authors have investigated this helicity in a simpler setup, using incoming polarized neutrons without subsequent polarization analysis (often called half-polarized experiment in the literature), in a geometry where $\mathbf{P}_{\mathbf{i}} \| \mathbf{Q}$. Figure 8 c shows the $\mathrm{L}$ scan profiles of magnetic satellites at $\mathbf{Q}=(4, \pm q, 1)$ for the ferroelectric state at $9 \mathrm{~K}$ which were measured after cooling in an electric field of $E= \pm 160 \mathrm{kV} / \mathrm{m}$. In the case of $P c>0$, the intensity of the satellite $(4,+q, 1)$ is approximately 9 times as high with neutron spin antiparallel $\left(I_{\downarrow}=I^{-+}+I^{--}\right)$to the scattering vector $\mathbf{Q}$ as that with neutron spin parallel $\left(I_{\uparrow}=I^{++}+I^{+-}\right)$. As for the satellite $(4,-q, 1)$, conversely, the contrary prevails. These behaviors are typical of a spiral magnet with a single helicity, where the spiral plane is almost perpendicular to the scattering vector. When the direction of the cooling electric field is reversed, the intensity $I_{\uparrow}$ of satellite $(4,+q, 1)$ becomes much stronger than $I_{\downarrow}$, demonstrating that the opposite helicity domain becomes dominant. The reversal of the ratio of $I_{\downarrow}$ to $I_{\uparrow}$ induced by the reversal of the electric field suggests that the spin helicity can be successfully controlled by a poling electric field.

In all we have seen up to now, we have completely neglected the incoherent scattering (isotopic and nuclear spin). The separation of this incoherent scattering from other sources of diffusion is another fruitful aspect of longitudinal polarization analysis, developed in the lecture of J.R. Stewart in the preceding Polarized Neutron Diffraction school [13].

Longitudinal polarization analysis, even though very powerful, suffers from some severe drawbacks. On the one hand, it only gives access to the diagonal terms of the polarization tensor define by Blume and Maleyev. The off-diagonal terms, which are very important for some problems (in particular they give a huge precision to determine small rotations of magnetic moments), cannot be measured. On the other hand, since only one component of the final polarization is analyzed, and the two remaining lost, 

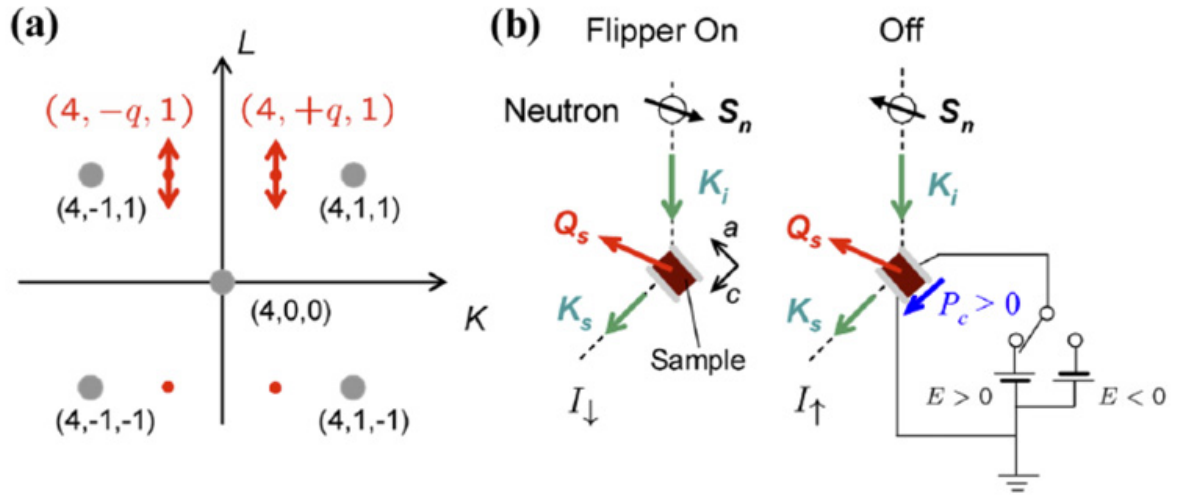

(c)

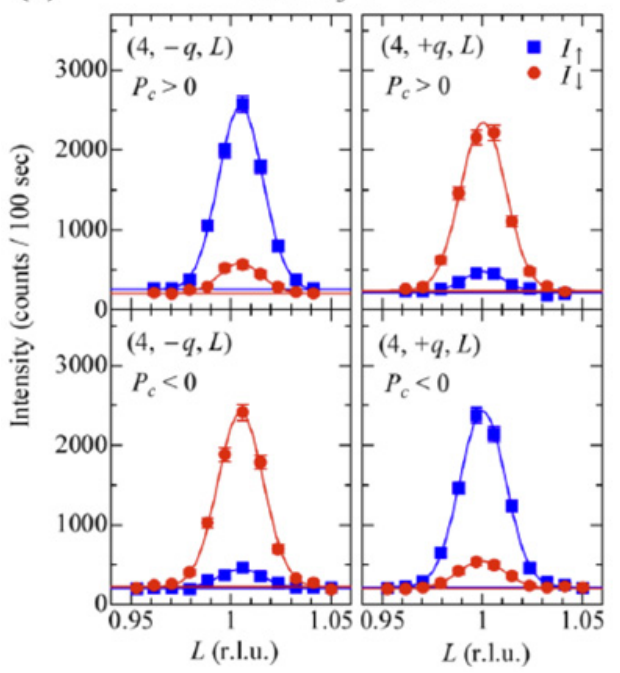

(d)

Counterclockwise Spiral

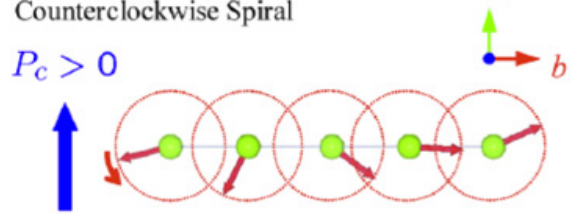

$\circ C$

Clockwise Spiral

$P_{c}<0$

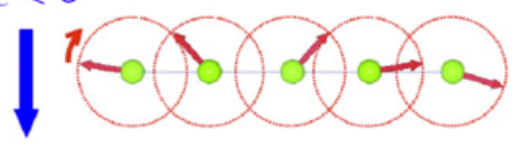

$\otimes C$

Figure 8. (a) L scans of magnetic satellites $(4, \pm q, 1)$ with $q \sim 0.27$. (b) The schematic illustration of the apparatus. The polarization of the neutron beam could be flipped by a spin flipper so as to be parallel (flipper off) or antiparallel (flipper on) to the scattering vector $\mathbf{Q}$. (c) Electric polarization dependence of intensity of magnetic satellites $(4, \pm q, 1)$ at $9 \mathrm{~K}$. L-scan profiles of magnetic satellites $(4, \pm q, 1)$ with $\mathrm{Pc}>0$ and $\mathrm{Pc}<0$. (d) The relation between the spin rotatory direction (or helicity) and the direction of electric polarization in $\mathrm{TbMnO}_{3}$. From Ref. [33].

it is impossible to distinguish a pure rotation of the polarization from a depolarization (due to domains for instance). A part of the information is lost, and as a consequence small effects are difficult to detect. Since the very beginning of the technique, it was clear that measuring all the terms of the tensor was highly desirable. But it took time, roughly twenty years, to technically reach this goal.

\section{SPHERICAL NEUTRON POLARIMETRY (SNP)}

Being able to have incident neutrons polarized in one direction and analyze them in another direction is a difficult task because of the strong influence of any magnetic field on the polarization vector. Indeed, such a task requires to be able to separate two magnetic guide fields with different directions by a zero field region, without any adiabatic rotation from one guide to the other. The solution to segment different magnetic regions on the neutron path has been found with Niobium Meissner superconducting 


\section{Collection SFN}
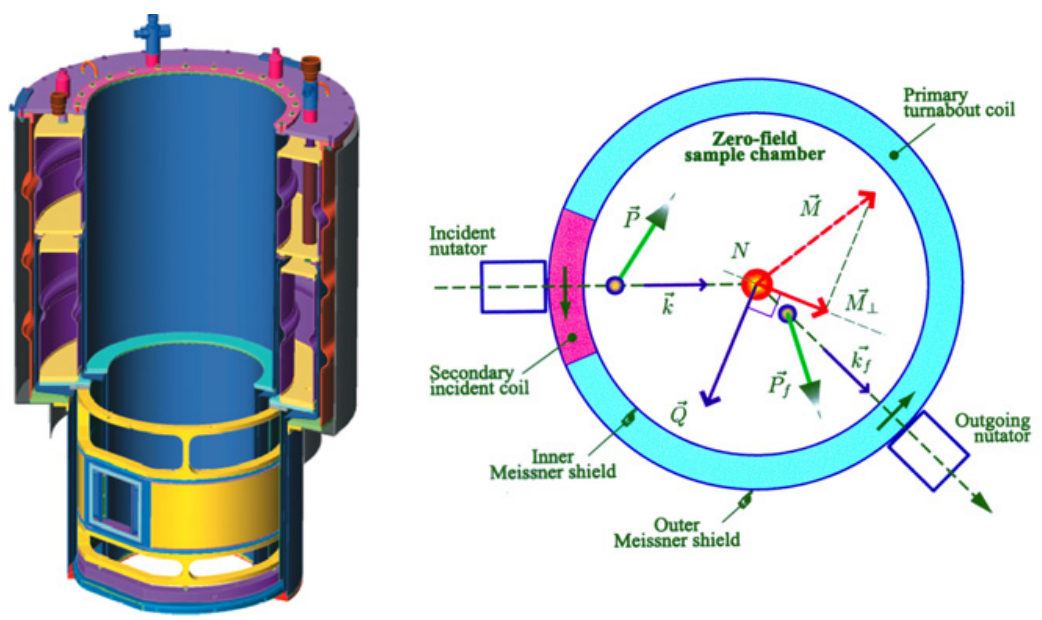

Figure 9. CRYOPAD.
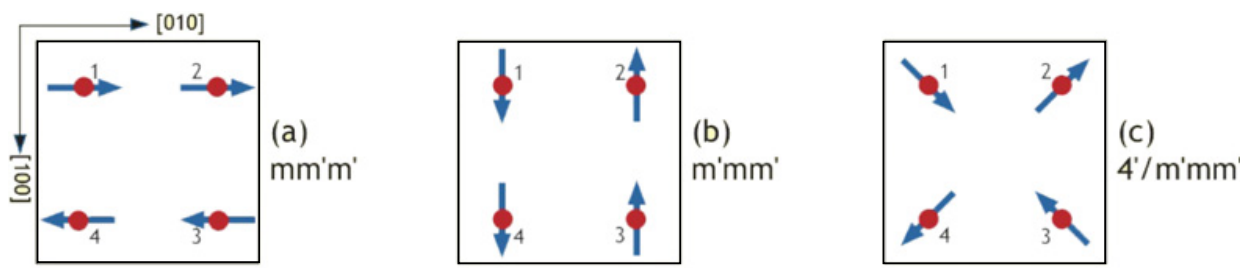

Figure 10. Three possible spin configurations of a tetragonal structure with $K=(0,0,1 / 2)$. From Ref. [13].

screens, as implemented in CRYOPAD (CRYogenic Polarization Analysis Device, see Fig. 9) [11]. Such screens are invaluable in initializing precise spin directions and keeping the neutron spin transition fully non adiabatic when going from one magnetic segment to the next. CRYOPAD makes use of two superconducting Meissner screens. Both screens have the form of long vertical tubes. The incident and scattered neutron beams pass through adiabatic spin-field rotators (nutators). Each nutator is a magnetic tunnel parallel to either the incident or scattered neutron beam and containing successively longitudinal and transverse static guide fields. The annular space between the two Meissner screens is large enough to house two coils. With such a configuration, it is possible to bring the incident polarization in any thought direction and analyze it in any other.

Since the first applications, spherical neutron polarimetry turned out to be extremely powerful and to be sometimes the only way to give definitive answers to pathological cases. In particular, to show how SNP is able to distinguish in some special cases between a canted and a collinear structure, let us consider a tetragonal structure with a propagation vector $\mathbf{K}=(0,0,1 / 2)$ and the spins in the (001) plane. Let us consider in addition that there are four magnetic atoms per unit cell: $\mathrm{A} 1$ at $(x, x, 0), \mathrm{A} 2$ at $(-x, x, 0), \mathrm{A} 3$ at $(-x,-x, 0), \mathrm{A} 4$ at $(x,-x, 0)$ with $x \approx 0.2$. The possible configurations of the plane at $z=0$ are shown in Figure 10. Calculating the magnetic structure factors, and the polarization matrices, it can be shown that the average intensity scattered by the two orthorhombic domains (a) and (b) is exactly the same as that scattered by the tetragonal structure (c). The diagonal terms of the polarization matrices are also the same, and only the off diagonal terms of the polarization matrices allows to distinguish between the two models. This means that polarization analysis at the LPA level could not give an answer, whereas SNP, because it measures off diagonal terms, is the only tool to decide between the two models. Calculations are developped in Ref. [13]. 

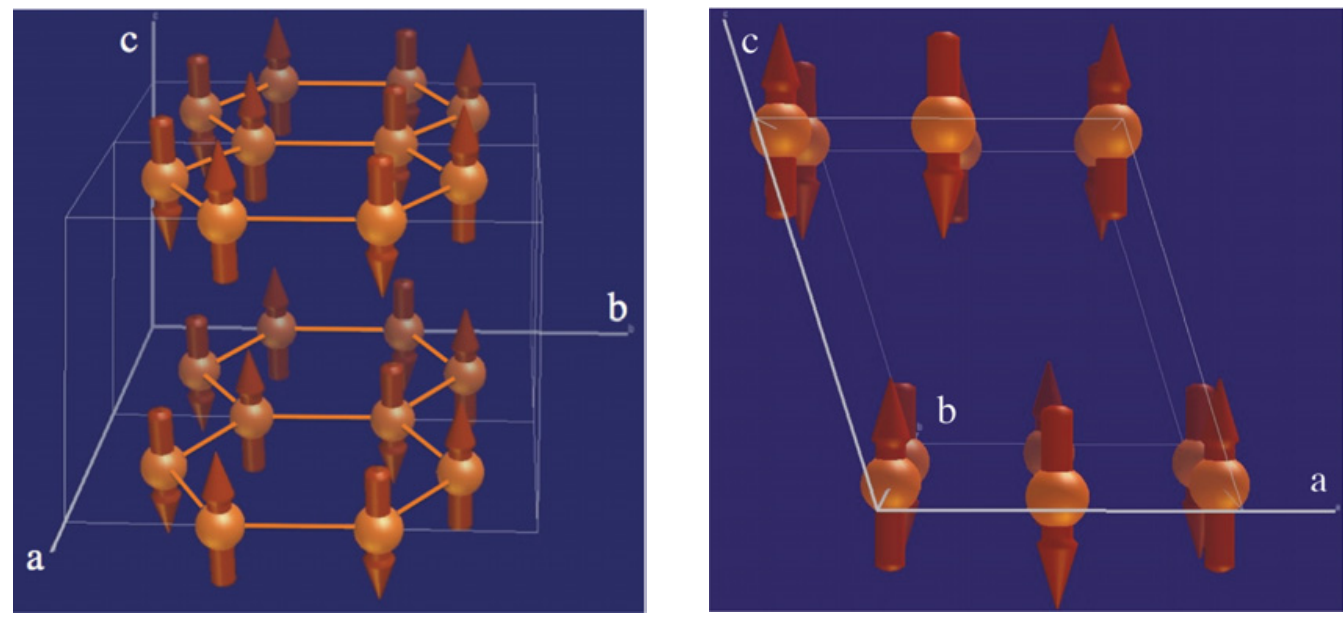

Figure 11. Proposed magnetic structure in $\mathrm{MnPS}_{3}$ from powder neutron diffraction [35].

To further illustrate the power of SNP, the case of $\mathrm{MnPS}_{3}$ is highly interesting [34]. This compound belongs to the thiophosphate family mainly studied for their intercalation properties. It is a lamellar compound, crystallizing in the monoclinic space group $\mathrm{C} 2 / \mathrm{m} . \mathrm{MnPS}_{3}$ is a highly resistive broad band semiconductor with a gap close to $3 \mathrm{eV}$ and is optically transparent with a green colour. The transition metal ions $\mathrm{Mn}^{2+}$ with spin 5/2, responsible for the magnetic properties, form a honeycomb lattice in the $(a, b)$ plane. It orders at $78 \mathrm{~K}$ in an antiferromagnetic collinear phase with a propagation vector $\mathbf{K}=\mathbf{0}$, in which each $\mathrm{Mn}$ is antiferromagnetically coupled with its first neighbor (Fig. 11). A first powder neutron experiment revealed that the moments were perpendicular to the $(a, b)$ planes, that is to the honeycomb lattice. However, because a few points were unclear, such as the anisotropy and the high ordering temperature, we decided to re-investigate this compound with both classical neutron diffraction and spherical neutron polarimetry on single crystal.

The crystal and magnetic structures were first checked on a four-circle diffractometer at low temperature. In a first step, the magnetic structure was refined from the collected integrated intensities. The resulting magnetic structure was found to agree with the published one. Below $\mathrm{T}_{N}$, the antiferromagnetic phase is characterized by a zero propagation vector. The four magnetic atoms have their magnetic moment along $c^{*}$ coupled following a +-+- sequence, i.e. each $\mathrm{Mn}^{2+}$ is antiferromagnetically coupled with its nearest neighbors in the $(a, b)$ plane, and the coupling between adjacent planes is ferromagnetic.

This magnetic structure description was further submitted to the stringent test of CRYOPAD. The polarization matrices measured for a set of $(0, k, l)$ reflections were refined. For each reflection, the 9 $P_{I J}$ and the $9 P_{-I J}$ terms (obtained by flipping the incident polarization) were recorded, to eliminate possible systematic errors $(I, J=X, Y, Z)$. The resulting observations were fitted using the magnetic structure described just above. The domain populations (two $180^{\circ}$ domains) and the $\mathrm{Mn}^{2+}$ magnetic moment amplitude were the only parameters of the fit. The result is shown in the first line of Figure 12 and is not very satisfactory. In a second step, the angle $\theta$ between the moment direction and the $c^{*}$ axis was let free to vary. After refinement, $\theta$ was found equal to $-8^{\circ}$ : the moments form an angle with respect to $c^{*}$ instead of being parallel to it (they are not perpendicular to the honeycomb lattice). The result is shown in the second line of Figure 12. This small change drastically improves the fit of the CRYOPAD data, whereas as far as unpolarized integrated intensities are concerned, the improvement is not obvious. The existence of a tilt of the magnetic moments with respect to natural symmetric directions 


\section{Collection SFN}
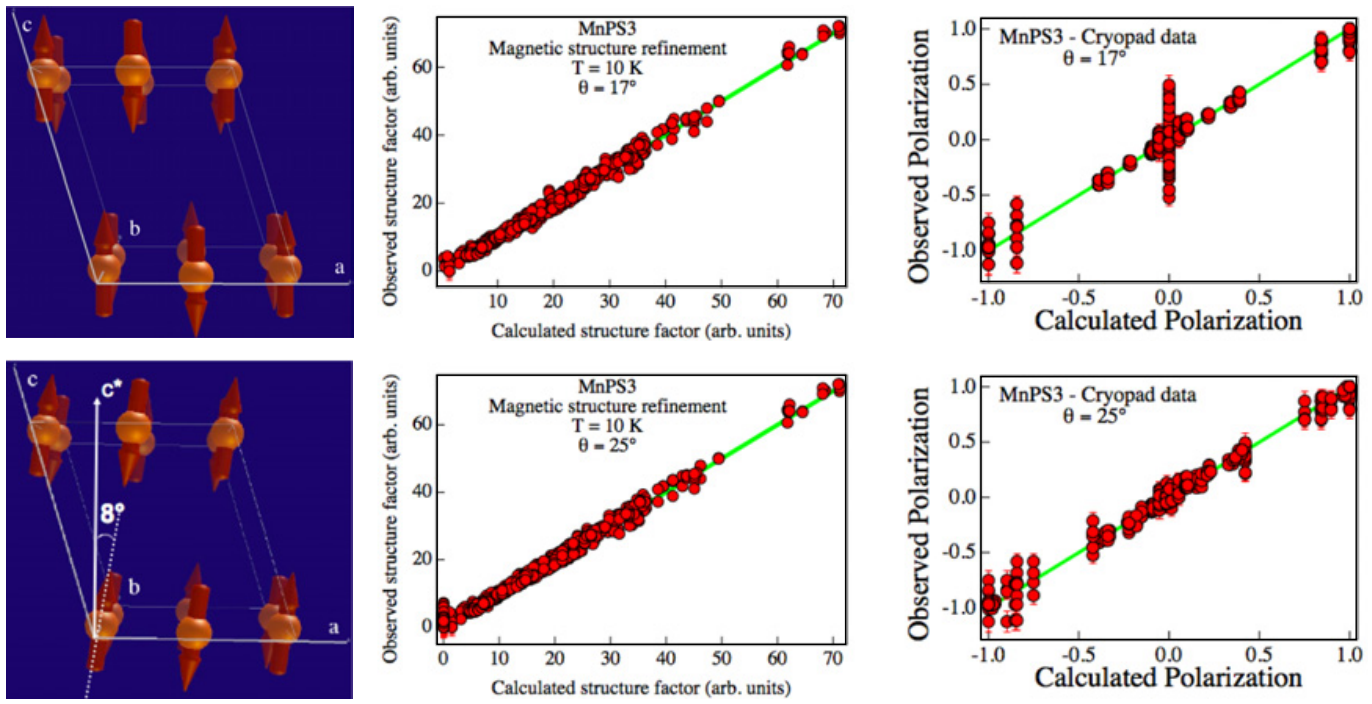

Figure 12. Comparison of two models of magnetic structure for $\mathrm{MnPS}_{3}$. From Ref. [34].

(crystal axes) indicates the presence of competing anisotropies. It could be due to dipolar and single-ion anisotropies. Without SNP, this tilt would certainly never had been evidenced.

In the case of $\mathrm{MnPS}_{3}$, SNP even allowed to go further. The observed magnetic structure is characterized by a zero propagation vector and loses the inversion center (the inversion center is combined with time reversal). The magnetic point group is thus $2 \% / \mathrm{m}$. This is of particular interest, because a linear magnetoelectric coupling is then allowed and the resulting magnetoelectric tensor is non-diagonal, with non-equal off diagonal terms. In the $(a, b, c)$ frame of the unit cell, this tensor is:

$$
\left(\begin{array}{ccc}
0 & \alpha_{12}^{M E} & 0 \\
\alpha_{21}^{M E} & 0 & \alpha_{23}^{M E} \\
0 & \alpha_{32}^{M E} & 0
\end{array}\right) .
$$

Given this possibility of linear ME effect, a way to characterize it is via the influence of a combined electric/magnetic field on the magnetic domain populations, those populations being accessible by spherical neutron polarimetry. Indeed, in such a magnetic structure, the nuclear and magnetic structure factors are in phase quadrature (real structural part, imaginary magnetic part). A spherical polarization analysis using CRYOPAD is able to give information on the respective proportion of the two $180^{\circ}$ antiferromagnetic domains. Calculations show that the matrix elements $P_{X Z}, P_{Z X}, P_{X Y}$, and $P_{Y X}$ are highly sensitive to the domain population parameter $\eta=\left(v_{1}-v_{2}\right) /\left(v_{1}+v_{2}\right)$, where $v_{1}$ and $v_{2}$ are the volume of the two domains (Fig. 13).

The $\mathrm{MnPS}_{3}$ crystal has therefore been submitted to combined electric and magnetic fields. The electric field, with voltages up to $2 \mathrm{kV}$, could only be applied along $c^{*}$, due to the lamellar sample shape. The tail of the cryostat was placed within the gap of an electromagnet delivering magnetic fields as high as $1 \mathrm{~T}$, parallel or perpendicular to the $E$ field direction i.e. along $c^{*}$ or $b^{*}$ respectively. Several ME annealings were produced by applying different electric and magnetic fields on the sample during cooling from the paramagnetic state $(90 \mathrm{~K})$ to the ordered phase $(50 \mathrm{~K})$. The fields were then switched off and the cryostat was positioned within CRYOPAD for the neutron polarization measurements. This procedure was repeated for each $(E, H)$ set. The domain populations were determined for increasing values of the product $E H$, for parallel or perpendicular relative orientations of $E$ and $H$. It turned out 


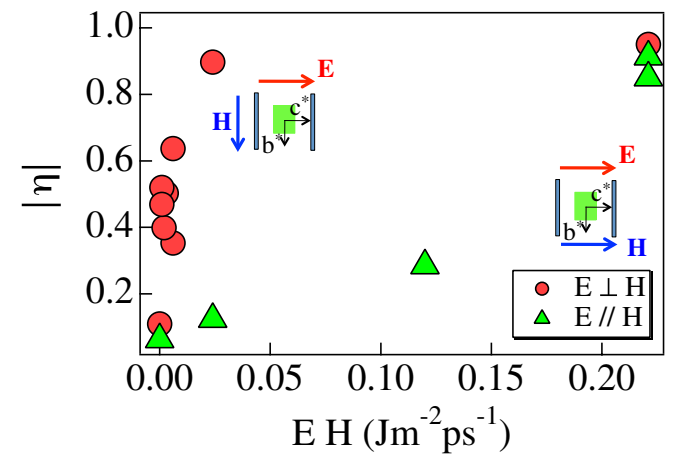

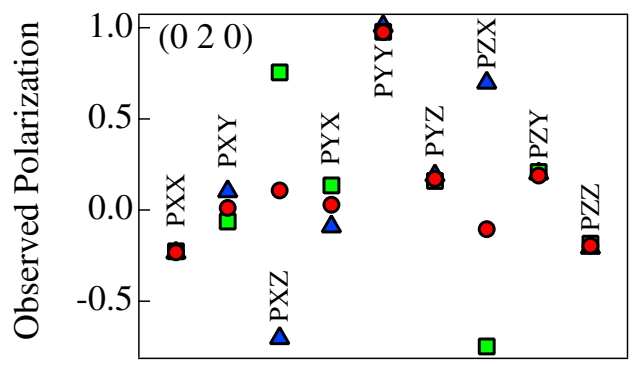

Figure 13. Left: absolute value of the relative domain populations $\eta$ as a function of the product of the electric and magnetic fields for different respective orientations. Right: polarization matrix elements of the $\left(\begin{array}{lll}0 & 2 & 0\end{array}\right)$ reflection for equi-populated antiferromagnetic domains (red dots) and for almost single domain 1 (blue triangles) or single domain 2 (green squares).

that the domain distribution could be modified from $\sim 50 \%$ of each domain without ME annealing to an almost single domain sample under the simultaneous action of $E$ and $H$ (Fig. 13). In the perpendicular geometry, the sample becomes rapidly single domain. A single domain was also unexpectedly achieved for the parallel geometry but for field products ten times larger. The observed imbalance of the domain populations in the parallel geometry can actually be explained by a misalignment of both fields of the order of $5^{\circ}$. The apparent dispersion of points for $E \perp H$ in Figure 13 is of the same origin. Note that the reversal of either $E$ or $H$ favors a selection of the other antiferromagnetic domain. Finally, it has been checked that no ME annealing was obtained with an electric field alone.

This experiment thus reveals the particular form of the linear ME tensor of $\mathrm{MnPS}_{3}$, in agreement with the symmetry of the magnetic space group: a non-diagonal linear magnetoelectric effect is clearly established in this quasi 2D antiferromagnet thanks to SNP. The ME domains population can be easily manipulated under reasonably weak fields through ME annealing.

As a last example of the power of SNP, we will now discuss the properties of the iron langasite compound $\mathrm{Ba}_{3} \mathrm{NbFe}_{3} \mathrm{Si}_{2} \mathrm{O}_{14}$ [36]. This material crystallizes in the non-centrosymmetric trigonal space group P321. The structure is chiral and it can be synthesized in two enantiomorphic species. The magnetic $\mathrm{Fe}^{3+}$ ions $(S=5 / 2)$ form a triangular array of triangles (trimer units) in the $(a, b)$ plane. From magnetization and specific heat measurements, a transition to an antiferromagnetic ordered phase was evidenced at $T_{N}=27 \mathrm{~K}$. A first powder neutron diffraction experiment showed the rise of extra Bragg peaks below $T_{N}$ which could be indexed with the propagation vector $\mathbf{K}=(0,0, \approx 1 / 7)$. The intensities of these new reflections yielded a $120^{\circ}$ arrangement of the magnetic moments in the $(a, b)$ plane inside a trimer unit. This $120^{\circ}$ arrangement is helically modulated in the perpendicular direction (along c). This magnetic structure is thus characterized by two kinds of magnetic chiralities, related to the sense of rotation of the spins inside the trimers (triangular chirality) on the one hand, and to the sense of rotation of the spins along the helix axis (helical chirality), in addition to the structural chirality [15]. The combination of the two magnetic chiralities gives four possibilities (Fig. 14).

The powder neutron diffraction experiment, because of the well known powder averaging, did not allow to distinguish between the different $120^{\circ}$ magnetic arrangements and to get information about the helical chirality. The four possible combinations account equally well for the diffractograms. Experiments on a single-crystal were necessary to go further. First, the structural chirality of the single-crystal used during the neutron experiments was determined by anomalous x-ray diffraction. This structural chirality has important consequences on the geometry of the magnetic exchange paths between the $\mathrm{Fe}^{3+}$ moments mediated by oxygen atoms. Given the structural chirality, unpolarized neutron diffraction on a single crystal shows that the ground state is compatible with two magnetic 


\section{Collection SFN}
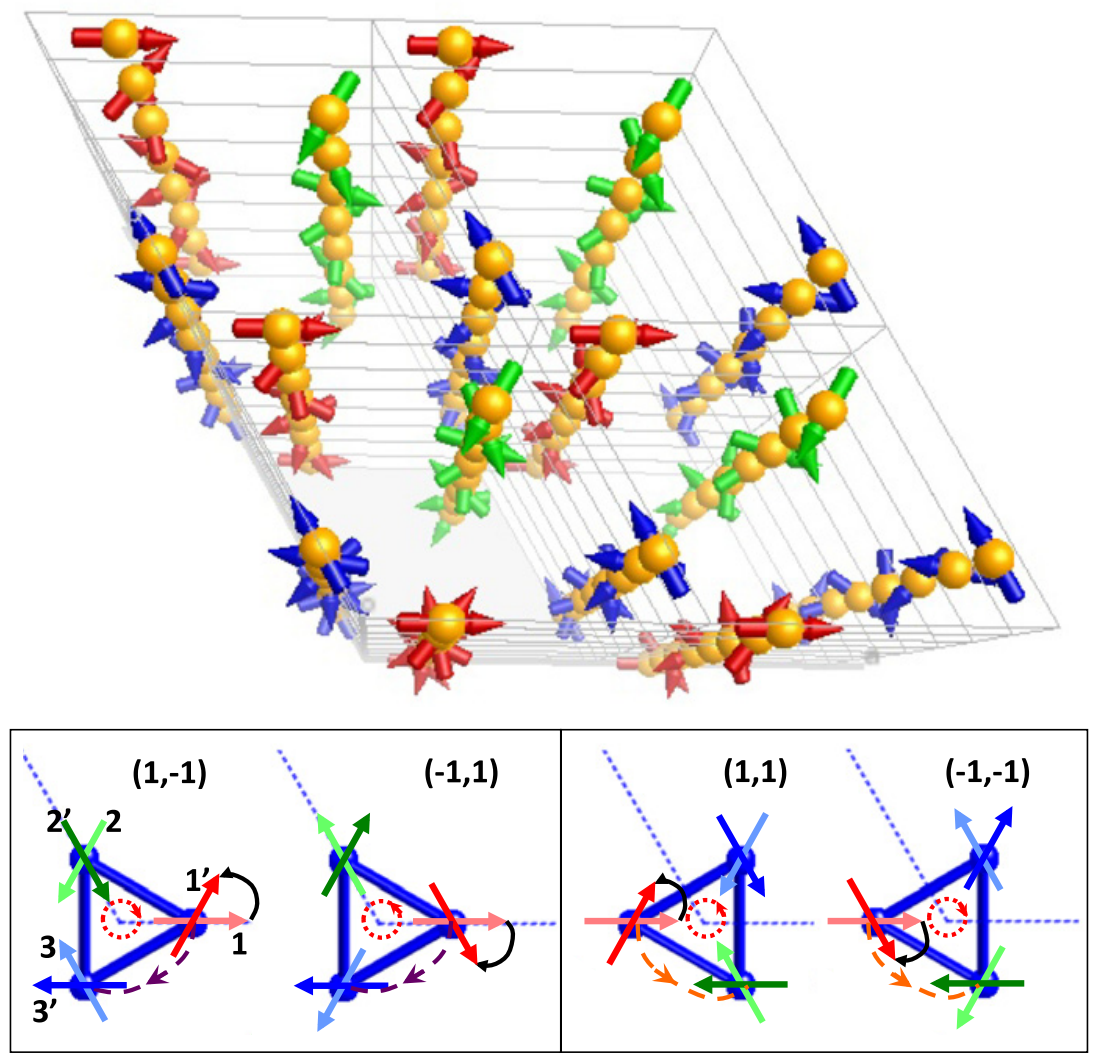

Figure 14. Top: magnetic structure of $\mathrm{Ba}_{3} \mathrm{NbFe}_{3} \mathrm{Si}_{2} \mathrm{O}_{14}$ with different colors for the three Bravais lattices of the trimer units. Bottom: representation of the magnetic structures associated with the 4 possible chiral ground states $( \pm 1, \pm 1)$.

chiralities out of the four possibilities (helical chirality $= \pm 1$, triangular chirality $= \pm 1$ ). The integrated intensities obtained on a crystal with a positive (resp. negative) structural chirality agree only with the $(+1,+1)$ or $(-1,-1)$ (resp. $(+1,-1)$ or $(-1,+1))$ chiral ground states. To be able to distinguish between these two possibilities, one has to turn to spherical neutron polarimetry, which proved the selection of one $(+1,+1)$ chirality couple, associated to the positive structural chirality. The same experiment was performed on a crystal with a negative structural chirality. This led to the opposite helical chirality, being thus compatible with the $(-1,+1)$ ground state. Here again, SNP turned out to be the only tool to conclude between the different models.

\section{CONCLUSION}

The aim of these lecture notes was to give a flavor of the fundamental aspects of polarized neutron diffraction, an overview of the practical details and to outline the unique information these techniques can bring. The high potentiality of this tool in solving condensed-matter physics problems justifies all the efforts devoted to the construction of spectrometers equipped with polarized neutron options and the efforts one should make to learn these techniques. In writing this chapter, the choice was to present only the basic equations and not to enter all the mathematical details. For people that want to go deeper in the subject, reference [13] is a very good starting point. 


\section{Acknowledgements}

I would like to thank V. Simonet and S. Petit for a critical reading of the manuscript and also all the organizers for this great school we had in Seignosse.

\section{References}

[1] C.G. Shull, J.S. Smart, Phys. Rev. 76 (1949), 1256.

[2] O. Halpern and M.H. Johnson, Phys. Rev. 51 (1937), 992.

[3] O. Halpern and M.H. Johnson, Phys. Rev. 52 (1937), 52.

[4] O. Halpern and M.H. Johnson, Phys. Rev. 55 (1939), 898.

[5] O. Halpern and T. Holstein, Phys. Rev. 59 (1941), 960.

[6] O. Halpern, M. Hamermesh and M.H. Johnson, Phys. Rev. 59 (1941), 981.

[7] M. Blume, Phys. Rev. 130 (1963), 1670.

[8] S.V. Maleyev, V.G. Bar'yakhtar and R.A. Suris, Sov. Phys. Solid State 12 (1963), 2533.

[9] R. Nathans, C.G. Shull, G. Shirane, A. Andresen, J. Phys. Chem. Solids 10 (1959), 138.

[10] R.M. Moon, T. Riste, and W.C. Koehler, Phys. Rev. 181 (1969), 920.

[11] F. Tasset, P.J. Brown and J.B. Forsyth, J. Appl. Phys. 63 (1988), 3606.

[12] F. Mezei, Zeit. Phys. 255 (1972) 146.

[13] Ecole thématique de la SFN, Neutrons Polarisés, ed. by N. Kernavanois, E. Ressouche, H. Schober, J.L. Soubeyroux, EDP Sciences (2005).

[14] L.C. Chapon, Private Comm.

[15] V. Simonet, M. Loire, R. Ballou, Eur. Phys.J. Special Topics 213, (2012) 5.

[16] P.J. Brown, Inter. Jour. Mod. Phys. B7, (1993) 3029.

[17] S.F. Gull and G.J. Daniell, Nature 272, (1978) 686.

[18] R.J. Papoular and B. Gillon, Europhys. Lett. 13, (1990) 429.

[19] E. Ressouche et al., to be published.

[20] J.X. Boucherle, J.Y. Henry, R.J. Papoular et al., Physica B 192, (1993) 25.

[21] P. Schleger, A. Puig-Molina, E. Ressouche et al., Acta Cryst. A53, (1997) 426.

[22] N.K. Hansen and P. Coppens, Acta Cryst. A34, (1978) 909.

[23] B. Gillon, C. Sangregorio, A. Caneschi et al., Inorganica Chimica Acta 360 (2007) 3802.

[24] W. Marshall and S.W. Lovesey, Theory of thermal neutron scattering, Clarendon press (Oxford), 1971.

[25] S.W. Lovesey, Theory of neutron scattering from condensed matter, Clarendon press (Oxford), 1983.

[26] G.H. Lander and T.O. Brun, J. Chem. Phys. 53, (1970) 1387.

[27] C. Stassis and H.W. Deckman, Phys. Rev. B 12, (1975) 1885.

[28] P.J. Brown, in International Tables for Crystallography, edited by A.J.C. Wilson, (Kluwer Academic Publishers, Dordrecht, 1992), 512.

[29] P.W. Anderson, in Magnetism, vol. 1, edited by G.T. Rado and H. Suhl (Academic Press, New York, 1963), 25.

[30] N. Kernavanois, E. Ressouche, P.J. Brown et al., J. Phys. Condens. Matter 15 (2003) 3433.

[31] A. Gukasov and P.J. Brown, J. Phys. Condens. Matter 14 (2002) 8831.

[32] I. Cabrera, M. Kenzelmann, G. Lawes et al., Phys. Rev. Lett. 103 (2009) 087201.

[33] Y. Yamasaki, H. Sagayama, T. Goto et al., Phys. Rev. Lett. 98 (2007) 147204.

[34] E. Ressouche, M. Loire, V. Simonet et al., Phys. Rev. B 82 (2010), 100408.

[35] K. Kurosawa, S. Saito, and Y. Yamaguchi, J. Phys. Soc. Jpn. 52 (1983) 3919.

[36] K. Marty, V. Simonet, E. Ressouche et al., Phys. Rev. Lett. 101 (2008) 247201. 